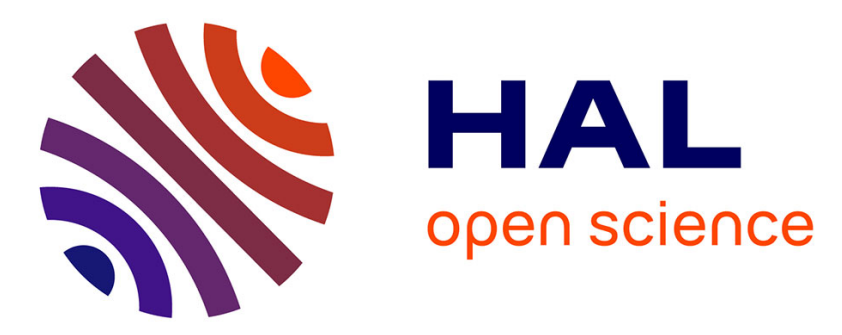

\title{
Impact de l'échoguidage sur les complications des cathéters veineux centraux par voie jugulaire chez les patients obèses en réanimation
}

\author{
Arianna Musi
}

\section{- To cite this version:}

Arianna Musi. Impact de l'échoguidage sur les complications des cathéters veineux centraux par voie jugulaire chez les patients obèses en réanimation. Médecine humaine et pathologie. 2015. dumas01277776

\section{HAL Id: dumas-01277776 https://dumas.ccsd.cnrs.fr/dumas-01277776}

Submitted on 23 Feb 2016

HAL is a multi-disciplinary open access archive for the deposit and dissemination of scientific research documents, whether they are published or not. The documents may come from teaching and research institutions in France or abroad, or from public or private research centers.
L'archive ouverte pluridisciplinaire HAL, est destinée au dépôt et à la diffusion de documents scientifiques de niveau recherche, publiés ou non, émanant des établissements d'enseignement et de recherche français ou étrangers, des laboratoires publics ou privés. 
UNIVERSITE DE PICARDIE JULES VERNE

FACULTE DE MEDECINE D'AMIENS

Année 2015

$N^{\circ} 2015-36$

\section{IMPACT DE L'ECHOGUIDAGE SUR LES COMPLICATIONS DES CATHETERS VEINEUX CENTRAUX PAR VOIE JUGULAIRE CHEZ LES PATIENTS OBESES EN REANIMATION.}

THESE POUR LE DOCTORAT EN MEDECINE (DIPLOME D'ETAT) PRESENTEE ET SOUTENUE PUBLIQUEMENT LE 22 AVRIL 2015 PAR

\section{Arianna MUSI}

Président du Jury :

Membres du Jury :

Directeur de Thèse :
Monsieur le Professeur Hervé DUPONT Madame le Professeur Claire ANDREJAK Monsieur le Professeur Emmanuel LORNE Monsieur le Docteur Yazine MAHJOUB Monsieur le Docteur Norair AIRAPETIAN 

A mon Président de Thèse

\section{Monsieur le Professeur Hervé DUPONT}

Professeur des Universités-Praticien Hospitalier

(Anesthésie-Réanimation)

Chef du service "Anesthésie-Réanimation"

Co-chef du Pôle "Anesthésie-Réanimation, médecine d'urgence"

Vous me faites l'honneur de présider ce jury.

Merci pour votre enseignement tout au long de ces années.

Soyez certain de mon profond respect. 

A mon Juge

\title{
Madame le Professeur Claire ANDREJAK
}

\author{
Professeur des Universités - Praticien Hospitalier \\ (Pneumologie)
}

Vous me faites l'honneur de juger ce travail, Permettez-moi de vous exprimer ma profonde reconnaissance. 

A mon Juge

\title{
Monsieur le Professeur Emmanuel LORNE
}

\author{
Professeur des Universités - Praticien Hospitalier
}

(Anesthésie-réanimation)

Merci de m'avoir fait l'honneur de juger ce travail.

Soyez assuré de ma profonde reconnaissance.

Merci pour votre enseignement. 

A mon Juge

\section{Monsieur le Docteur Yazine MAHJOUB}

Maître de Conférences des Universités

Praticien Hospitalier Anesthésiologie Réanimation, médecine d'urgence

Merci de m'avoir fait l'honneur de juger ce travail.

Tu as toujours su te rendre disponible pour répondre à mes questions. Merci pour ton enseignement et ta gentillesse. 

A mon Directeur de Thèse

\title{
Monsieur le Docteur Norair AIRAPETIAN
}

\author{
Praticien Hospitalier \\ Réanimation Chirurgicale
}

Je te remercie profondément pour m'avoir fait confiance et pour m'avoir guidée à travers ce travail de longue haleine. Je n'oublierai pas tout ce que tu as fait pour moi, soyez en assuré! 



\section{A tous les médecins,}

\section{A tout le personnel,}

des services d'Anesthésie et Réanimation d'Amiens, Beauvais, Compiègne, Creil et Château-Thierry :

mes sincères remerciements.

\section{A mes co-internes :}

merci de m'avoir supportée pendant ces longues années. Quels bons souvenirs je garderai de ces cinq ans ensemble!

\section{A Julie :}

ma colloc', mon binôme, mon amie. Du temps a passé depuis nos premiers trajets de train vers la Picardie côte à côte! Malgré la distance, nous saurons garder notre complicité, j'en suis sûre!

\section{A mes amis :}

ma vie va redevenir un peu plus normale, nous allons pouvoir en profiter!

\section{A ma belle-famille :}

merci pour votre gentillesse et pour m'avoir accueillie si chaleureusement dans votre famille.

\section{A mes parents :}

c'est à vous en premier que je dédie ce diplôme, à vous qui m'avez donné toutes les possibilités et qui m'avez guidée et soutenue dans tous mes choix. J'aspire à ce que vous soyez toujours fiers de moi. Vi voglio un bene infinito.

\section{A Mathieu :}

merci d'avoir été là, à chaque instant. 



\section{LEXIQUE}

CVC : cathéters veineux centraux

SFAR : société française d'Anesthésie-Réanimation

RFE : recommandations formalisées d'experts

TVP : thrombose veineuse profonde

OMS : organisation mondiale de la santé

IMC : indice de masse corporelle

SDRA : syndrome de défaillance respiratoire aiguë

$\mathrm{CHU}$ : centre hospitalier universitaire

$\mathrm{MHz}$ : méga Hertz

$\mathrm{cm}$ : centimètre

$\mathrm{Kg}$ : kilogramme

$\mathrm{m}$ : mètre

UFC : unité formant une colonie

$\mathrm{ml}:$ millilitre

ILC : infection liée au cathéter

$\mathrm{HC}$ : hémoculture

$\mathrm{mm}:$ millimètre

$\min :$ minute

TP : temps de prothrombine

TCA : temps de céphaline activé

CCPPRB : comité consultatif de protection des personnes dans la recherche biomédicale

IGS : indice de gravité simplifié

DS : déviation standard

$\mathrm{n}$ : nombre de patients

$\mathrm{j}:$ jours

vs : versus

NICE : national intitute of clinical excellence 

TABLE DES MATIERES

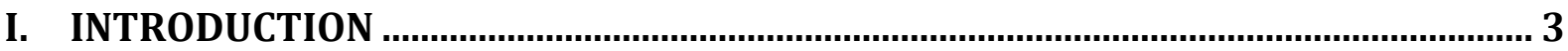

1. Anatomie des vaisseaux et la répercussion de ses variations ......................................... 3

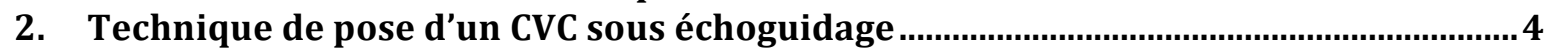

3. Complications des cathéters veineux centraux

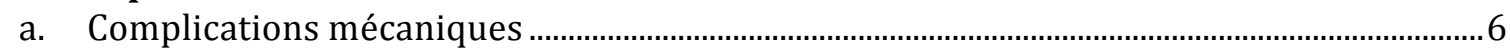

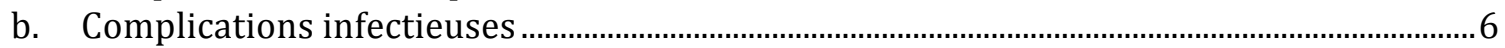

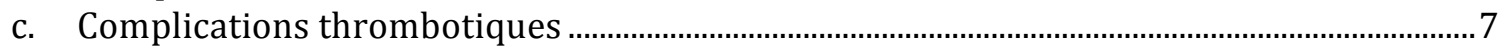

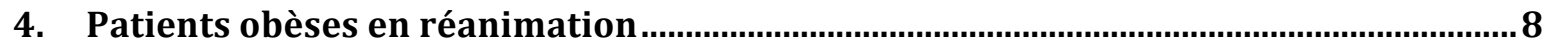

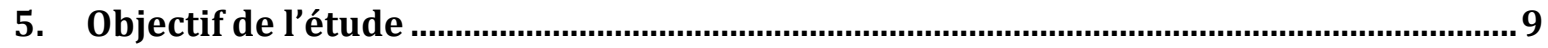

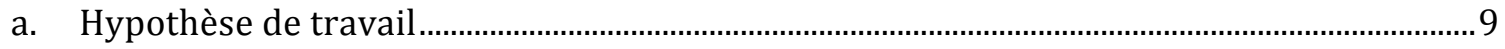

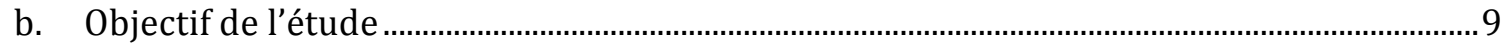

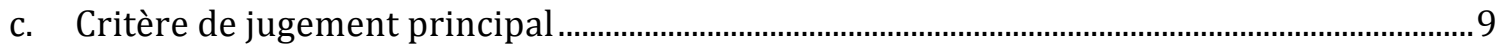

d. Critères de jugement secondaires ..................................................................................................

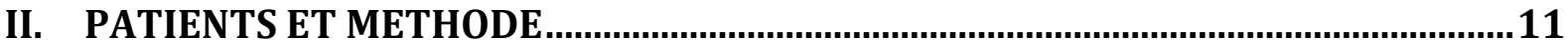

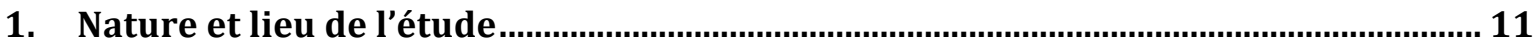

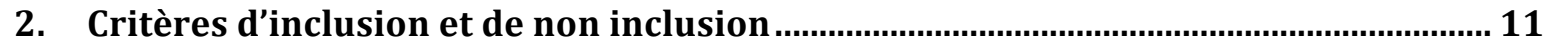

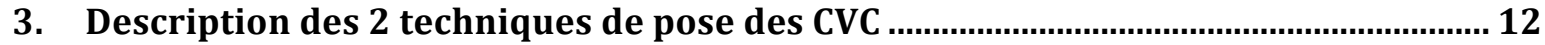

a. Technique de pose par repérage anatomique ................................................................................12

b. Technique de pose par échoguidage ……………................................................................... 12

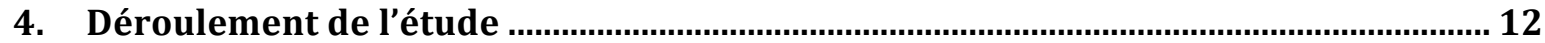

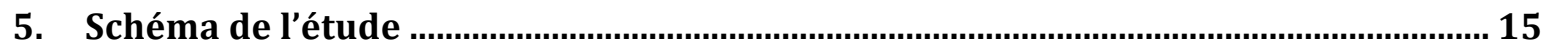

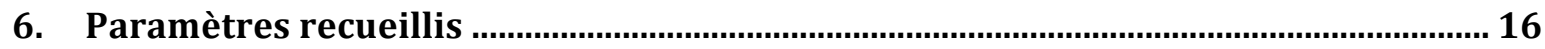

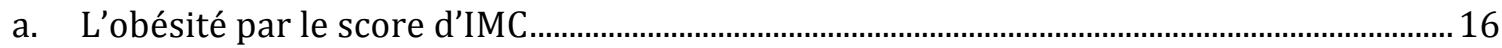

b. Le critère de jugement principal ............................................................................................ 16

c. Les critères de jugement secondaires ..................................................................................... 17

d. Les données démographiques et cliniques à l'inclusion .........................................................18

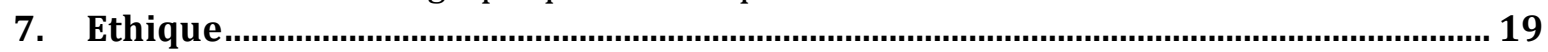

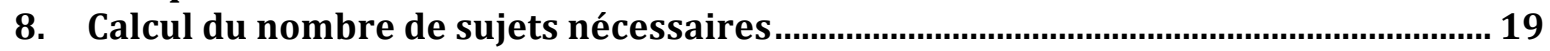

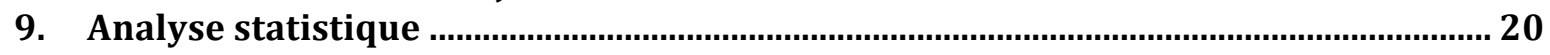

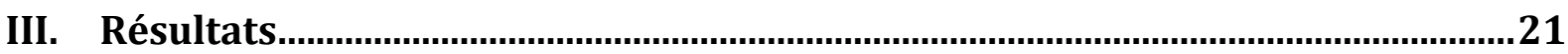

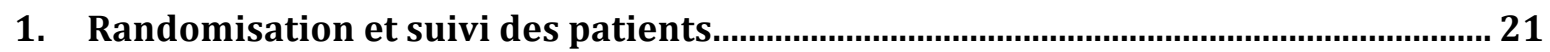

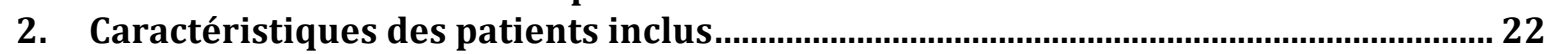

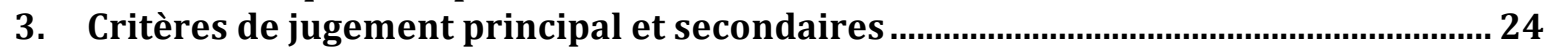

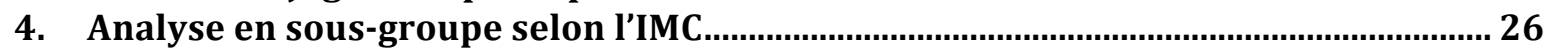

5. Analyse en sous-groupe selon de l'expérience de l'opérateur .................................... 28

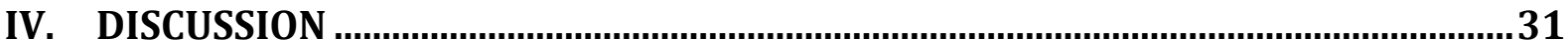

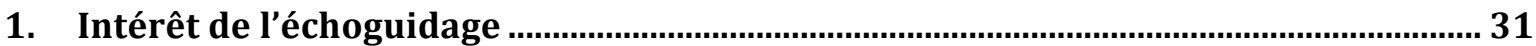

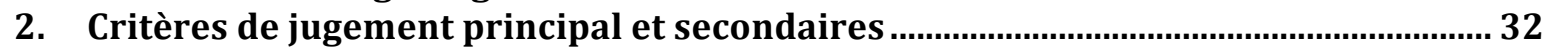

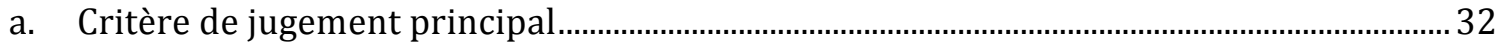

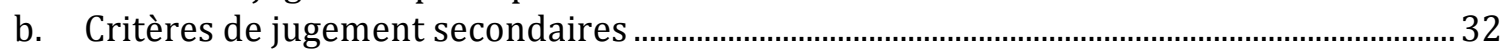

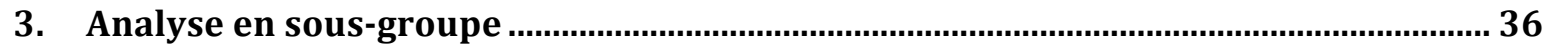

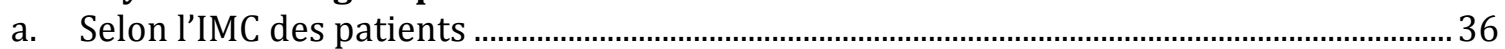

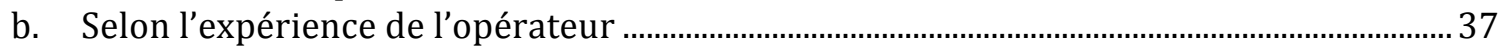

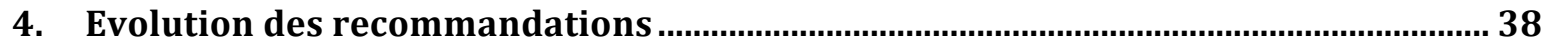

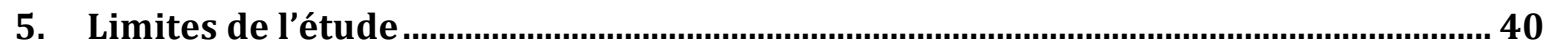

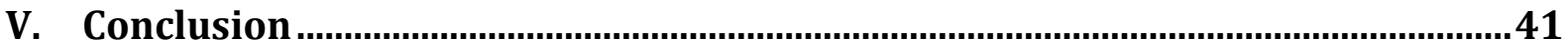




\section{INTRODUCTION}

Les cathéters veineux centraux (CVC) sont des dispositifs intravasculaires insérés au niveau de la veine jugulaire interne, sous-clavière ou fémorale.

Ils permettent, entre autres, la mesure de la pression veineuse centrale, un remplissage vasculaire rapide lorsque l'utilisation d'un abord périphérique est impossible, la perfusion continue d'amines vasopressives ou encore de produits veinotoxiques (par exemple, la nutrition parentérale ou encore certains antibiotiques).

La mise en place des CVC, qu'ils soient posés pour une courte ou longue durée, représente l'un des gestes les plus fréquents en réanimation. En effet dans les années 1990 on estimait à cinq millions le nombre de CVC mis en place chaque année aux Etats-Unis [1] et en Europe les études suggèrent qu'actuellement $50 \%$ à $60 \%$ des patients en réanimation seraient porteurs d'un cathéter veineux central [2,3].

L'insertion des CVC s'est faite pendant des décennies en se basant sur des repères anatomiques de surface qui, en fonction de différents contextes, notamment l'obésité, peuvent être difficiles à visualiser, voire chez certains patients carrément absents. La position des veines centrales peut être atypique ou bien les veines peuvent être thrombosées, anormales ou trop petites pour qu'il soit possible de les cathétériser.

Bien qu'étant un acte très fréquent, la pose d'un CVC n'en est néanmoins pas un geste dénué de iatrogénie, parfois sévère. La morbidité immédiate est principalement imputée aux complications mécaniques, mais il existe aussi des complications tardives comme l'infection et la thrombose.

C'est dans ce contexte et dans le but de diminuer la morbidité directement imputable aux CVC que sont apparues les premières études utilisant l'échographie.

\section{Anatomie des vaisseaux et la répercussion de ses variations}

Dans la région cervicale l'artère carotide interne se trouve entourée d'un paquet vasculo-nerveux composé de deux éléments : la veine jugulaire interne et le nerf vague. Par rapport à cette artère, la veine jugulaire interne se trouve généralement en position antérieure et latérale. Dans le travail de Gordon et coll., elle est dans cette position classique dans 70\% des cas pour ce qui concerne la veine jugulaire interne droite, et dans $66 \%$ des cas pour la veine jugulaire gauche [4]. 
Dans l'étude de Denys et coll., la veine jugulaire interne était dans 92\% des cas dans sa position classique, dans 3\% des cas elle était virtuelle, sans ponction ni cathétérisation possible même après des manœuvres de Valsalva, et enfin dans 5\% elle se trouvait en dehors de sa position habituelle [5].

Dans ces nombreux cas de figure, la position réelle des vaisseaux n'est pas identifiable par les seuls repères anatomiques de surface.

Par ailleurs, chez $62,5 \%$ des patients inclus dans le travail de Lichtenstein et coll. , il existe une asymétrie entre les veines jugulaires internes concernant leur diamètre respectif, avec une prédominance de la veine jugulaire droite [6]. Il a été montré que, comparativement à celle de la jugulaire interne gauche, la pose d'un CVC à droite comportait un taux de succès supérieur avec des complications moindres [7]. Pour expliquer ce résultat il avait été mis en avant que la majorité des opérateurs étaient droitiers et se trouvaient donc mieux exposés en posant le cathéter à droite. Mais l'asymétrie entre les veines jugulaires peut elle aussi contribuer à ce résultat.

Un dernier élément vient s'ajouter, le fait que le trajet de la veine jugulaire interne droite soit linéaire vers la veine cave supérieure, alors que la veine jugulaire interne gauche se jette dans la veine sous-clavière avec un angle de $90^{\circ}$, rendant plus difficile sa cathétérisation.

\section{Technique de pose d'un CVC sous échoguidage}

Les premières études se servant des ultrasons dans la pose des CVC utilisaient le mode Doppler ou le mode bidimensionnel.

Les premiers travaux s'intéressant à la technique de pose des CVC en s'aidant du mode Doppler ont débuté dans les années 1980.

Le mode doppler permettait de distinguer la position d'une veine ou d'une artère par le son qu'elle émettait. Avec ce mode, une veine est distinguée par son débit continu et un son grave rythmé par le temps respiratoire. Une artère, elle, est reconnue par son débit pulsatile et un son aigu rythmé par la fréquence cardiaque $[8,9]$.

Dans une première méta-analyse de 1996, les auteurs ont montré que l'utilisation du mode Doppler, pour repérer en temps réel la localisation des vaisseaux et le bon positionnement du cathéter, permettait de réduire le taux de complications et d'améliorer le succès des CVC en position jugulaire interne et sous-clavière [10].

Cependant, l'efficacité du mode Doppler pour le repérage en temps réel semblait être très opérateur-dépendante [11], et ne s'observait qu'après une longue durée d'apprentissage. 
Tout ceci a conduit progressivement à l'abandon de cette technique au profit du mode bidimensionnel $[12,13]$.

Le mode bidimensionnel ouvre le champ à deux techniques différentes :

- l'échorepérage, qui consiste en un marquage du site de ponction après repérage échographique des structures vasculaires,

- l'échoguidage, qui consiste à ponctionner et cathétériser la veine sous contrôle échographique continu, avec une vision en temps réel de l'anatomie vasculaire.

De nombreuses études menées dans les unités de réanimation [14-16] et dans les services d'urgences [17-19], ont montré la supériorité de la technique par échoguidage par rapport à la technique utilisant les repères anatomiques seuls. De même, la technique par échorepérage semble plus performante par rapport à celle par ponction « en aveugle » [20, $21]$.

En s'intéressant plus spécifiquement au mode bidimensionnel, la technique par échoguidage semble être plus performante que la technique par échorepérage, que ce soit au sujets des échecs ou des complications engendrées [16, 21-23].

De nombreux pays, notamment les Etats-Unis et le Royaume Unis, recommandent d'utiliser la technique d'échoguidage pour la pose des CVC [24-27].

Malgré la mise en place des ces recommandations, seulement 15 à $41 \%$ des médecins utilisent en première intention la technique de pose par échoguidage [28-31].

L'absence d'un appareil d'échographie peut se révéler, à lui tout seul, un facteur limitant. Par ailleurs, la technique d'échoguidage nécessite un apprentissage technique, même si celui-ci est qualifié de simple et rapide par l'étude de Nguyen et coll., réalisée chez les praticiens non expérimentés [32]. La principale raison évoquée par les médecins sur la non utilisation de l'échoguidage est qu'ils ne jugent pas nécessaire de s'en servir pour diminuer le taux d'échecs et la morbidité associée.

Enfin l'hypothèse de la non connaissance de ces recommandations écrites en langue étrangère a poussé la Société Française d'Anesthésie-Réanimation (SFAR) à publier en Décembre 2014 des Recommandations Formalisées d'Experts (RFE), pour inciter les praticiens français à faire rentrer la technique de pose des CVC par échoguidage dans leur pratique courante [33]. 


\section{Complications des cathéters veineux centraux}

a. Complications mécaniques

Les principales complications mécaniques lors de la pose d'un cathéter veineux central en position jugulaire interne sont la ponction artérielle, l'hématome et le pneumothorax. Leur fréquence globale est estimée entre $5 \%$ et $19 \%$ [34]. Plus spécifiquement le taux de complications à type de ponction artérielle est évalué à $10 \%$ et presque à $3 \%$ celui de l'hémothorax ou du pneumothorax [35].

Comme nous le montrent Schummer et coll. dans leur étude, il n'y a pas de corrélation entre un faible niveau d'expérimentation et la survenue de ce type de complications. Sur 1794 procédures réalisées par des opérateurs expérimentés uniquement, les mêmes pourcentages étaient retrouvés: $3 \%$ d'échecs et environ 12\% de complications ou malpositions [36].

L'apport de l'échographie sur ce type de complications tient sur le fait qu'elle permet de détecter les variations anatomiques éventuelles, la présence d'une veine thrombosée ou encore de petit calibre.

\section{b. Complications infectieuses}

Les différentes voies de contamination sont les suivantes :

- par voie cutanée, la plus fréquente, par défaut d'asepsie lors de la pose du CVC ou par infection secondaire du site d'insertion,

- par voie endoluminale, due le plus fréquemment aux manipulations septiques des raccords, elle devient le mécanisme prépondérant après 3 semaines d'utilisation d'un CVC,

- par voie hématogène, plus rare.

A l'origine du mécanisme de colonisation se trouve le dépôt d'un film de protéines et de plaquettes, qui favorise l'adhésion des bactéries sur le dispositif intravasculaire. Les microorganismes produisent le « slime », qui facilite leur adhésion.

Chaque année aux Etats-Unis on recense 80000 bactériémies imputables aux CVC [37], responsables d'environ 28000 décès annuels dans les services de réanimation. 
Comme le prouvent Pronovost et coll., l'incidence de ces bactériémies décroît si l'on respecte certaines recommandations de bonnes pratiques lors de la pose et de l'entretien des CVC, comme le choix d'un site de pose en position sous-clavière par rapport au site jugulaire interne et fémoral [38]. D'autres études aussi privilégient la voie sous-clavière pour son moindre risque infectieux [3, 39].

Selon Deshpande et coll., il n'existe pas de différence en terme de complications infectieuses sur les trois sites d'insertion des CVC, mais seulement si les conditions de pose sont optimales et gérées par un médecin sénior [40]. De même qu'une revue récente de la littérature ne retrouve pas de différence entre les trois sites de pose en terme de bactériémie liée au CVC [41].

Selon l'étude Cathédia, le taux de complications infectieuses à type de colonisation du cathéter de dialyse par voie jugulaire interne se chiffre à $25 \%$ [42].

Sans échographie, le taux de complications infectieuses imputables à un CVC est généralement compris entre 5 et $26 \%$ [34]. Malheureusement, la littérature est pauvre au sujet de l'apport de l'échographie dans ce secteur.

\section{c. Complications thrombotiques}

Les patients hospitalisés dans les services de réanimation possèdent des facteurs de risque multiples de développer une maladie thromboembolique veineuse, tels que l'immobilisation, la déshydratation, les troubles de la coagulation. Le diagnostic d'une thrombose veineuse profonde (TVP) n'est pas aisé, à la fois par la difficulté des patients en réanimation à signaler les éventuels symptômes, et par le fait qu'elles sont le plus souvent silencieuses.

L'incidence des TVP en réanimation est estimée à 30\% en absence de prophylaxie médicamenteuse, et variant entre 10 à 34\% malgré l'administration d'une prophylaxie [4348]. La présence d'un dispositif intravasculaire comme un CVC augmente ce risque.

Fabiani et coll. montrent que cathétériser la veine jugulaire interne avec l'introducteur d'un cathéter de Swan-Ganz, même pour une courte durée, augmente le risque de thrombose [49].

Dans l'étude Cathédia, parmi les 75 patients porteurs d'un cathéter de dialyse en position jugulaire interne, l'incidence de la complication thrombotique était de $22,7 \%$ [42]. 
Il n'y a pas de données dans la littérature évaluant le potentiel emboligène des complications thrombotiques des $\mathrm{CVC}$, mais elles sont à considérer comme toute autre TVP avec leur morbidité potentielle associée.

\section{Patients obèses en réanimation}

Selon l'Organisation mondiale de la santé (OMS), 35\% des adultes dans le monde sont atteints d'obésité ou de surpoids.

Les complications associées à l'obésité, notamment le diabète et les maladies cardiovasculaires, sont responsables d'au moins 2,8 millions de décès par an [50].

En France, l'obésité concernait $15 \%$ des adultes en 2012, contre seulement 6,1\% en $1980[50]$

Le diagnostic de l'obésité passe notamment par le calcul de l'indice de masse corporelle (IMC), méthode qui reste à ce jour le seul moyen simple pour estimer la masse grasse d'un individu [51]. Il correspond au poids (en $\mathrm{kg}$ ) divisé par le carré de la taille (en mètres). Selon la classification de l'OMS, on parle de surpoids lorsque l'IMC est supérieur à $25 \mathrm{~kg} / \mathrm{m} 2$ et d'obésité lorsqu'il dépasse $30 \mathrm{~kg} / \mathrm{m} 2$.

Cette croissance de l'obésité dans la population générale entraîne parallèlement une augmentation des patients obèses en réanimation, dont leur prévalence est estimée entre 9 et $26 \%$ des patients de réanimation médicochirurgicale [52].

L'obésité est associée à une augmentation de la morbidité en réanimation [53]. Parmi les études qui retrouvent une augmentation de la mortalité chez les obèses par rapport aux non obèses, un taux plus élevé de sepsis, de pneumopathie nosocomiale, de syndrome de détresse respiratoire aiguë (SDRA), d'infection sur cathéter, d'insuffisance cardiaque contribuerait à l'aggravation du pronostic en cas d'obésité [54-56].

Anatomiquement, la profondeur des vaisseaux est corrélée à l'IMC. Or, plus le vaisseaux est profond, plus l'angle de ponction est important et sa cathétérisation difficile. De plus chez le patient obèse il y a une perte des repères anatomiques externes, ce qui majore la difficulté des abords vasculaires percutanés.

Il existe peu ou pas d'études s'étant intéressées spécifiquement à l'impact du cathétérisme veineux échoguidé dans la population représentée par les patients obèses en réanimation. 


\section{Objectif de l'étude}

a. Hypothèse de travail

Nous émettons l'hypothèse que, au sein de la population représentée par les patients obèses en réanimation, le taux de complications (mécaniques, infectieuses et thrombotiques) liées à l'insertion d'un cathéter veineux central en position jugulaire interne pourrait être réduit grâce à l'utilisation de la technique de pose par échoguidage.

\section{b. Objectif de l'étude}

L'objectif de cette étude est de démontrer que le taux de complications (mécaniques, infectieuses et thrombotiques), liées à l'insertion d'un CVC en position jugulaire interne chez les patients obèses en réanimation, est diminué par l'utilisation de la technique de pose par échoguidage par rapport à celle par simple repérage anatomique.

c. Critère de jugement principal

Notre critère de jugement principal est un critère composite, caractérisé par la survenue d'une complication mécanique et/ou infectieuse et/ou thrombotique secondaire au cathétérisme veineux central, comparativement entre deux techniques : l'échoguidage et le repérage anatomique.

\section{d. Critères de jugement secondaires}

Comparer entre les deux techniques :

- les taux de complications mécaniques (surtout l'échec, la ponction artérielle, l'hématome)

- les taux de complications infectieuses (colonisation, infection liée au cathéter)

- les taux de complications thrombotiques

- le temps de pose

- le nombre de ponction

- la mortalité 


\section{PATIENTS ET METHODE}

\section{Nature et lieu de l'étude}

Il s'agit d'une étude clinique prospective, randomisée, monocentrique, réalisée au sein de 4 services de Réanimation du Centre Hospitalier Universitaire d'Amiens :

- Réanimation Chirurgicale, Professeur Dupont

- Réanimation Neurochirurgicale, Professeur Dupont

- Réanimation Médicale, Professeur Slama

- Réanimation Cardio-Vasculaire, Professeur Dupont

Chaque unité de réanimation a procédé à la désignation d'un investigateur local qui était responsable des inclusions et du recueil des données. Ces dernières ont ensuite été recueillies et traitées par l'investigateur principal qui en a fait l'analyse.

\section{Critères d'inclusion et de non inclusion}

\section{Critères d'inclusion :}

- Age $>18$ ans

- Nécessité de la pose d'un CVC en jugulaire interne

- $\mathrm{IMC}>30 \mathrm{~kg} / \mathrm{m}^{2}$

- Consentement signé du patient

\section{Critères de non inclusion :}

- Pas de critères 


\section{Description des 2 techniques de pose des CVC}

a. Technique de pose par repérage anatomique

Après repérage de l'artère carotide voisine de la veine jugulaire interne, par simple palpation du pouls, cette dernière était ponctionnée par la voie de Boulanger, et le cathéter introduit par la méthode de Seldinger modifiée [57].

b. Technique de pose par échoguidage

L'échographe utilisé était de type SiteRite ${ }^{\circledR} V$ équipé d'une sonde multifréquences de 5-10 MHz $\left(\operatorname{Bard}^{\circledR}\right)($ annexe 1).

La première étape était d'isoler la sonde d'échographie par une protection plastique stérile. Puis il fallait équiper cette sonde d'un « ergot» porte-aiguille, qui permette d'adapter l'aiguille de ponction, afin de les solidariser et d'en faciliter la ponction veineuse. Nous avions le choix entre 3 tailles d'ergots possibles $(1,5,2,5$ et 3,5 cm), en fonction de la profondeur de la lumière du vaisseau à atteindre. Information directement disponible sur l'écran de l'échographe (annexe 2).

Ensuite on appliquait un gel stérile sur la zone de ponction qui permettait de visualiser les structures anatomiques.

La veine jugulaire interne était ponctionnée par la voie de Boulanger sous contrôle échographique continu.

Le cathéter était, dans cette méthode aussi, introduit par la méthode de Seldinger modifiée [57].

\section{Déroulement de l'étude}

Dans chaque unité de réanimation participant à l'étude nous avons présenté le protocole de l'étude, ainsi que le matériel et les modalités de recueil des données.

Les indications de pose et d'ablation des CVC étaient des décisions médicales, prises de façon indépendante de l'étude. 
Aucune mesure de nature à modifier la pose ou le retrait des cathéters, ni à modifier les habitudes de pansement ou de maintenance des cathéters n'était imposée.

Dès lors que l'indication de la pose d'un CVC était décidée, une randomisation permettait d'inclure le patient soit dans le bras «échoguidage », soit dans le bras « repères anatomiques ».

Cette randomisation était rendue possible grâce à un logiciel mis à disposition sur un serveur intranet et disponible depuis toutes les unités de réanimation.

L'ensemble des cathéters veineux centraux inclus dans l'étude a été inséré par des séniors ou médecins réanimateurs en formation, ayant reçu au préalable une initiation à la technique avec échoguidage par une expérience de deux poses de CVC minimum.

En ce qui concerne le conditionnement du patient avant la pose d'un cathéter veineux central, il était nécessaire que le patient soit surveillé par électrocardioscope. En effet lors de la pose au niveau jugulaire interne, le guide métallique est suffisamment long pour arriver au niveau des cavités cardiaques et peut produire des extrasystoles ou salves de tachycardie ventriculaire, qui peuvent être mal tolérées par le patient.

Les conditions d'asepsie et la préparation du matériel ont été celles habituellement réalisées et recommandées lors de la pose d'un dispositif intravasculaire. On effectuait une désinfection cutanée par la procédure qui suit :

- nettoyage de la zone d'insertion à la polyvidone aqueuse à 4\% moussante (Bétadine $\left.\operatorname{Scrub}^{\circledR}\right)$,

- rinçage à l'eau stérile puis séchage avec des compresses stériles,

- première application de polyvidone iodée à $5 \%$ alcoolique (Bétadine ${ }^{\circledR}$ alcoolique) débordant largement la zone d'insertion (15 à $20 \mathrm{~cm})$.

L'opérateur, après un lavage chirurgical des mains, devait porter une charlotte, un masque, une blouse et des gants stériles.

Ensuite il délimitait la zone d'insertion du CVC par des champs stériles et enfin procédait à une deuxième application de polyvidone iodée à $5 \%$ alcoolique (Bétadine ${ }^{\circledR}$ alcoolique).

Enfin il préparait le matériel nécessaire sur un champ stérile : une aiguille de ponction montée sur une seringue, et le guide métallique. 
Les cathéters centraux posés (de marque Arrow $^{\circledR}$ ), étaient simple, double, ou triple lumière (16 ou $20 \mathrm{~cm}$ de longueur). Ce choix aussi était laissé libre à l'opérateur en fonction de l'indication de l'utilisation du CVC (mono lumière par exemple pour la simple perfusion d'antibiotiques, jusqu'à triple lumière lorsque chez le même patient il était nécessaire d'administrer simultanément un remplissage vasculaire rapide, des catécholamines et une nutrition parentérale).

L'opérateur pouvait choisir sans distinction le coté d'insertion du CVC, en fonction de sa préférence, ou par défaut si un autre accès veineux était présent en controlatéral ou encore afin de réduire au maximum la morbidité pour le patient (par exemple, il est préférable de poser un CVC en jugulaire interne du même coté d'un drain thoracique en aspiration).

Afin d'exposer au mieux le site de ponction le patient était positionné avec les bras le long du corps, la tête légèrement en extension et tournée légèrement du coté opposé au site de ponction. On inclinait le lit de $15^{\circ}$ en position de Trendelenburg pour améliorer le remplissage veineux et diminuer le risque d'embolie gazeuse.

Avant de procéder à la ponction de la veine jugulaire, une anesthésie locale au point de ponction des tissus sous cutanés était réalisée par de la Lidocaïne ${ }^{\circledR}$ non adrénalinée $1 \%$ ou $2 \%$.

Quelle que soit la technique de pose utilisée, le cathéter était finalement fixé à la peau par deux nœuds chirurgicaux et recouvert par un pansement occlusif.

Lorsque la décision médicale de l'ablation du cathéter était prise, celui-ci était mis directement en culture selon la technique décrite par Brun-Buisson, qui consiste à couper stérilement l'extrémité distale du cathéter sur une longueur d'environ 4 à $5 \mathrm{~cm}$, pour en effectuer l'analyse bactériologique [58].

La durée d'inclusion de chaque patient dans le protocole de l'étude correspondait à la durée de mise en place du cathéter, en y ajoutant les 4 jours suivants, pendant lesquels un écho doppler du trajet vasculaire jugulaire interne concerné devait être effectué, pour rechercher des signes de thrombose veineuse. 


\section{Schéma de l'étude}

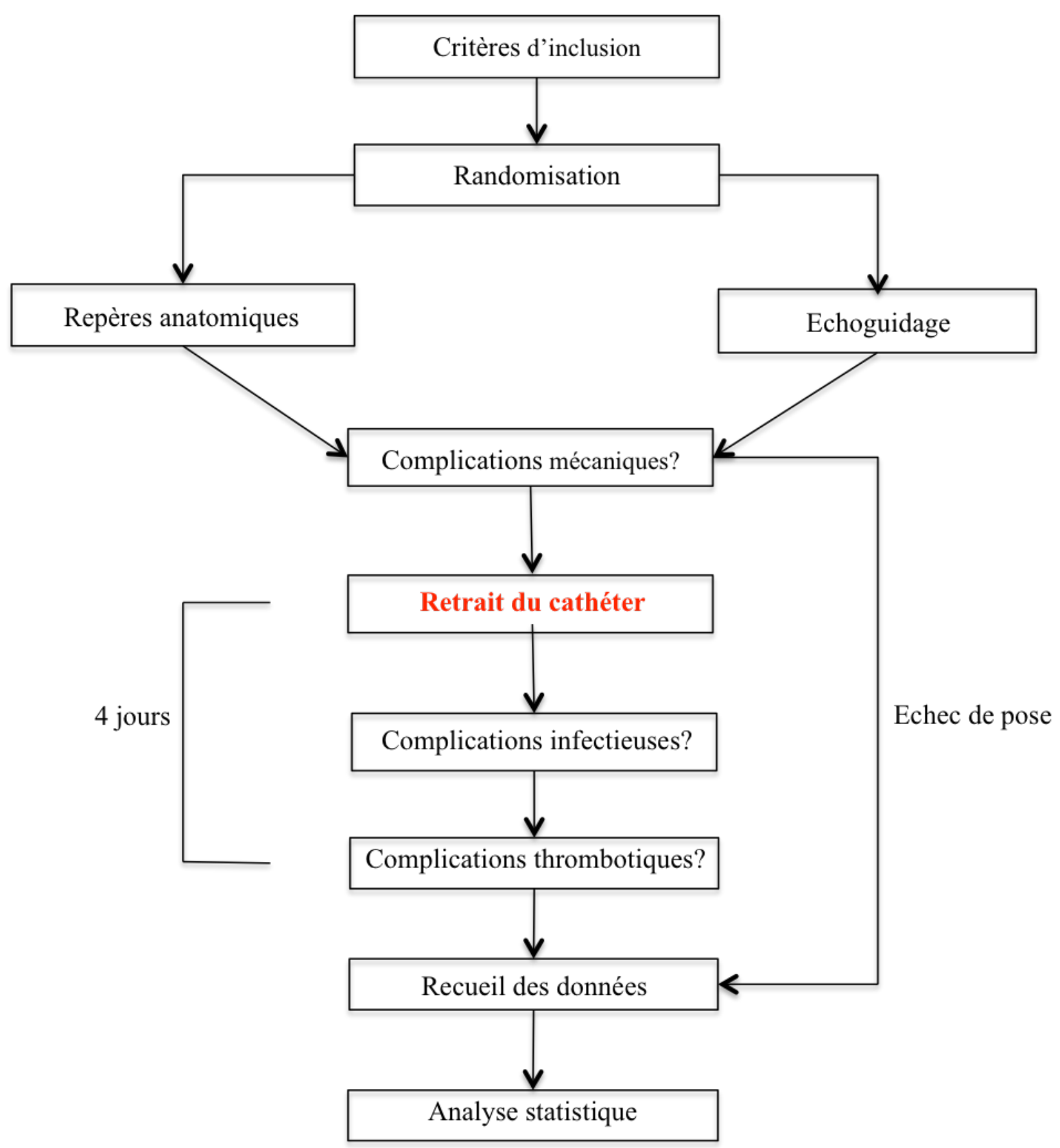




\section{Paramètres recueillis}

a. L'obésité par le score d'IMC

L'obésité, définie par un score d'IMC $\geq 30 \mathrm{Kg} / \mathrm{m}^{2}$, était l'un des critères d'inclusion indispensables. Le score d'IMC étant défini par la formule suivante [59]:

$$
\operatorname{IMC}\left(\mathrm{kg} / \mathrm{m}^{2}\right)=\text { Poids }(\mathrm{Kg}) / \text { Taille }^{2}(\mathrm{~m})
$$

L'obésité est divisée en trois classes de gravité croissante : modérée (IMC entre 30 et $34,9 \mathrm{Kg} / \mathrm{m}^{2}$ ), sévère (IMC entre 35 et $39,9 \mathrm{Kg} / \mathrm{m}^{2}$ ) et morbide (IMC supérieur ou égal à 40 $\left.\mathrm{Kg} / \mathrm{m}^{2}\right)[51,54]$.

Le score d'IMC de chaque patient au moment de la pose du CVC était relevé, sans devoir rendre compte d'une éventuelle variation de poids par rapport au poids de référence du patient lors de son séjour en réanimation (notamment imputable à une inflation hydro-sodée par exemple), ou de la répartition entre masse maigre et grasse.

\section{b. Le critère de jugement principal}

Le critère de jugement principal était défini comme un critère composite, regroupant l'ensemble des complications mécaniques, infectieuses et thrombotiques décrites ci-dessous.

Dès lors que l'une d'entre elles était relevée, le critère de jugement principal était considéré comme présent.

- les complications mécaniques à la pose regroupent :

- l'échec : défini par un nombre de ponctions cutanées $\geq 3$ à l'insertion du cathéter, - la ponction artérielle,

- l'hématome nécessitant une compression, - le pneumothorax. 
- les complications infectieuses regroupent [60]:

- la colonisation du cathéter :

définie par une culture positive avec un seuil $\geq 10^{3} \mathrm{UFC} / \mathrm{ml}$ selon la méthode décrite par Brun-Buisson [58],

- l'infection liée au cathéter (ILC) :

en absence de bactériémie : elle est définie par l'association d'une colonisation du CVC avec une régression partielle ou totale des signes infectieux dans les 48 heures qui suivent, ou par la présence d'un aspect purulent de l'orifice d'entrée du CVC ou encore par la présence d'une tunnelite.

en présence de bactériémie : elle est définie par l'association d'une hémoculture (HC) positive (deux en cas de Staphylococcus epidermidis) survenant dans les 48 heures encadrant l'ablation du CVC avec :

soit une culture positive du site d'insertion au même germe soit avec une colonisation du CVC positive au même germe soit un rapport quantitatif entre $\mathrm{HC}$ centrales/périphériques $>5$ soit un rapport différentiel entre $\mathrm{HC}$ centrale/périphérique $>2$ heures

- $\quad$ les complications thrombotiques regroupent :

Dans les 4 jours suivant l'ablation du CVC, un écho doppler veineux était réalisé sur le trajet vasculaire concerné à la recherche d'une perte de compressibilité du segment veineux.

Le diamètre antéro-postérieur du thrombus était alors mesuré. Seuls les thrombus dont le diamètre antéro-postérieur mesuré dépassait $5 \mathrm{~mm}$ étaient considérés comme à l'origine d'une complication thrombotique.

c. Les critères de jugement secondaires

- les complications mécaniques et/ou infectieuses et/ou thrombotiques décrites ci-dessus, - le temps de pose du CVC, entre la première effraction cutanée de l'aiguille de ponction jusqu'au retour veineux présent au niveau de la première voie du cathéter,

- le nombre de ponctions cutanées avant la pose définitive,

- la mortalité. 


\section{d. Les données démographiques et cliniques à l'inclusion}

- $\quad$ les données démographiques :

- l'âge,

- le sexe,

- le motif d'entrée en réanimation,

- le poids, la taille,

- les antécédents d'hypertension artérielle, de diabète, d'insuffisance rénale chronique (définie par une clairance de la créatinine inférieure à $60 \mathrm{ml} / \mathrm{min} / 1,73 \mathrm{~m}^{2}$ pendant plus de trois mois, dialysée ou non), d'immunodépression (cancer, hémopathie maligne, éthylisme chronique, cirrhose),

- le score de Mc Cabe qui tenait compte d'une maladie chronique sous-jacente et de l'appréciation de son pronostic dans les trois mois qui précédaient l'hospitalisation en réanimation. Trois groupes de pronostics différents étaient constitués [61] (annexe 4).

- $\quad$ les données cliniques :

- le recours à la ventilation mécanique

- la température, la pression artérielle moyenne, la fréquence cardiaque

- l'Indice de Gravité Simplifié II (IGS II) qui permet l'évaluation de la gravité globale des patients hospitalisés en unité de soins intensifs (USI) ou en réanimation (annexe 3),

- l'administration de catécholamines, d'une antibiothérapie (probabiliste ou adaptée), d'un traitement anticoagulant (préventif ou curatif),

- les taux de globules blancs, de plaquettes, le temps de prothrombine (TP), de céphaline activé (TCA). 


\section{Ethique}

En 2010 le Comité Consultatif de Protection des Personnes dans la Recherche Biomédicale (CCPPRB) de la région Nord Ouest a approuvé le protocole et le document de consentement éclairé, et donné son accord pour la surveillance du déroulement de l'étude et le réexamen à intervalles réguliers.

Le consentement éclairé pour cette recherche biomédicale avec bénéfice individuel direct était requis auprès du malade lui-même. Dans le cas particulier de cette étude, effectuée en situation d'urgence et lorsque le recueil du consentement par le patient était impossible, l'accord était demandé en premier lieu à la personne de confiance, sinon $\mathrm{au}(\mathrm{x})$ membre(s) de la famille présent(s). Il était de la responsabilité de l'investigateur d'obtenir le consentement éclairé de chaque patient, ainsi que les signatures appropriées du document de consentement éclairé, avant la mise en route de toute procédure de l'étude.

Le document de consentement éclairé expliquait au patient, en termes simples et avant son entrée dans l'étude, quels étaient les risques et les bénéfices. Dans ce document figurait une déclaration que le consentement était libre, que le patient avait pris connaissance des risques et des bénéfices que présentait sa participation à l'étude, et qu'il était libre de sortir de l'étude à tout moment.

Le CHU d'Amiens, en tant que promoteur de l'étude, a souscrit une assurance pour tous les patients et services participants.

Les procédures médicales de cet essai étaient conformes aux recommandations les plus récentes de la déclaration d'Helsinki et de la loi n $88-1138$ du 20 décembre 1988 modifiée et ses décrets d'application relative à la protection des personnes qui se prêtent à des recherches biomédicales.

\section{Calcul du nombre de sujets nécessaires}

Le calcul du nombre de sujets nécessaires est basé sur le plan de l'étude randomisée. A partir des données de la littérature, on évalue que l'inclusion de 304 patients permettrait de montrer une baisse des complications de $40 \%$ à $25 \%$, avec un risque de première espèce bilatéral de $5 \%$ et une puissance de $80 \%$. Cette estimation repose sur les études de CVC posés chez des patients non obèses réalisées jusqu'à ce jour. 
En tenant compte de 2 analyses intermédiaires à $1 / 3$ et $1 / 2$ de l'effectif total, il aurait fallu 310 patients au total. En prenant en compte un pourcentage de 10\% de patients non évaluables pour le critère principal, le nombre de patients à inclure dans l'étude était de 346 au total.

La durée prévisible de l'étude était initialement estimée à 24 mois à dater de l'accord du CCPPRB du CHU d'Amiens. Cependant, du fait de la vitesse d'inclusion modérée (les patients admis en réanimation possèdent souvent un cathéter veineux central mis au préalable par exemple dans l'unité de déchoquage du service d'accueil des urgences ou encore au bloc opératoire), et des résultats attendus fortement en faveur du groupe échoguidage, nous avons prévu de réaliser deux analyses intermédiaires, à $1 / 3$ puis à $1 / 2$ des effectifs, afin d'optimiser la durée totale de l'étude tout en gardant une significativité statistique pour les résultats obtenus.

\section{Analyse statistique}

Les variables sont exprimées sous forme de moyenne \pm écart-type ou de proportion selon qu'il s'agit de données quantitatives ou qualitatives.

Les comparaisons entre les groupes comportant les variables quantitatives ont été réalisées à l'aide d'un test t de Student.

Concernant les variables nominatives, le test $\mathrm{du} \chi^{2}$ a été appliqué pour la comparaison des 2 groupes, avec une éventuelle correction de Yates ou un test exact de Fischer pour les petits échantillons.

Selon la méthode de l'alpha spending function [62] avec des bornes fixées avec la méthode d'O'Brien et Fleming [63], les critères statistiques de supériorité du bras « échoguidage » par rapport au bras « repères anatomiques » étaient respectivement de $\mathrm{p}<0.0026, \mathrm{p}<0.011$ et $\mathrm{p}<0.04558$ à la $1^{\text {ère }}$ analyse intermédiaire, à la $2^{\text {ème }}$ analyse intermédiaire et à la fin de l'étude.

L'analyse statistique a été réalisée grâce au logiciel SPSS version 17.0 (SPSS inc). 


\section{Résultats}

\section{Randomisation et suivi des patients}

Les inclusions ont eu lieu de Juin 2010 à Février 2015, date à laquelle l'analyse intermédiaire à la moitié des effectifs a été réalisée et que le critère statistique de supériorité du bras « échoguidage » par rapport au bras « repères anatomiques » a été atteint.

$\mathrm{Au}$ total 220 patients ont été randomisés, 109 patients dans le groupe «repères anatomiques », et 111 patients dans le groupe « échoguidage ».

Parmi eux, 44 patients sont sortis de l'étude, les principales raisons étant l'absence de données concernant la mise en culture du CVC après son retrait et la non réalisation de l'écho doppler de contrôle dans les quatre jours suivants, soit par cause de décès du patient, soit parce qu'il a été perdu de vue. Les autres motifs d'exclusion de l'étude sont décrits dans la figure 1.

Le terme « non ponctionné » signifie que le patient a été randomisé, mais que la pose du CVC n'a pas eu lieu selon les modalités décrites par l'étude. Il s'agissait essentiellement de la présence d'anomalies anatomiques (thrombose de la veine jugulaire, ligature du tronc inominé imposant un site de pose du CVC autre que la veine jugulaire interne).

$\mathrm{Au}$ total, 176 patients ont été inclus dans l'analyse de notre étude, 90 patients dans le bras « repères anatomiques », et 86 patients dans le bras « échoguidage ».

Figure 1 : Randomisation et suivi des patients

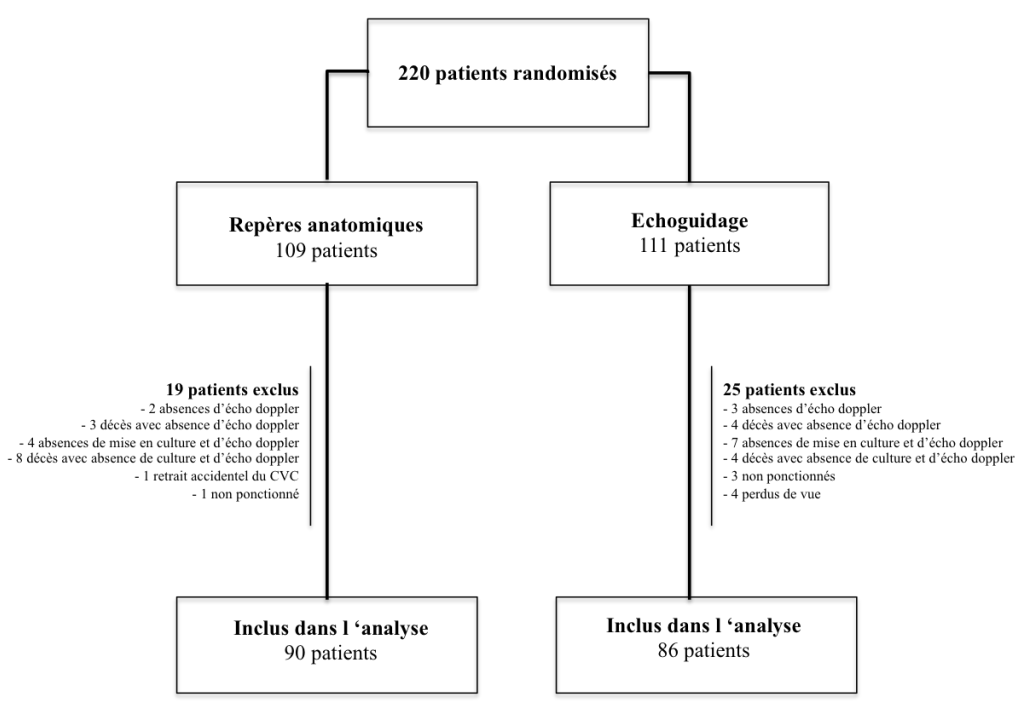




\section{Caractéristiques des patients inclus}

Les caractéristiques des patients inclus et leur répartition dans chaque bras de randomisation sont décrites dans le tableau 1 .

Dans la population générale l'âge moyen est de $61 \pm 15$ ans, le score d'IGS II moyen de $50 \pm 15$ avec $60 \%$ d'hommes, l'IMC moyen est de $38 \pm 7 \mathrm{Kg} / \mathrm{m}^{2}$, avec une mortalité moyenne de $17 \%$ en réanimation. Parmi eux, $66 \%$ étaient atteints d'hypertension artérielle, $36 \%$ de diabète, $17 \%$ d'immunodépression et enfin $8 \%$ d'insuffisance rénale chronique.

Les motifs d'entrée en réanimation étaient répartis de la façon suivante :

- défaillance hémodynamique : $28 \%$,

- défaillance respiratoire : $22 \%$,

- prise en charge post-opératoire de péritonite : $14 \%$,

- prise en charge post-opératoire de chirurgie réglée : $11 \%$,

- polytraumatisme : $7 \%$,

- insuffisance rénale aigue : $2 \%$

- arrêt cardio-respiratoire, pancréatite aigüe, dissection aortique, endocardite, intoxication médicamenteuse volontaire : 16\%

La moitié des patients recevait un traitement prophylactique de la maladie thromboembolique veineuse. Pour $29 \%$ des patients il s'agissait d'un traitement curatif, alors que chez les $21 \%$ restants, aucune forme d'anticoagulation n'était administrée.

La majorité des patients était sous antibiothérapie au moment de la pose du cathéter, ce qui représente un pourcentage de $82 \%$ des patients. L'antibiothérapie était pour $66 \%$ d'entre eux probabiliste et pour les $34 \%$ restants adaptée à un antibiogramme.

L'indication du CVC était dans 64\% la perfusion de produits veinotoxiques, dans $24 \%$ le défaut de voie d'abord périphérique, et enfin dans $12 \%$ le changement d'un accès central précédemment inséré.

La durée de vie moyenne des cathéters était de $12 \pm 10$ jours.

Parmi les motifs de retrait du CVC, dans $38 \%$ des cas il n'y avait plus d'indication au cathéter, dans $31 \%$ des cas il s'agissait d'une suspicion d'infection, dans 19\% des cas d'un retrait systématique, dans $6 \%$ des cas d'un dysfonctionnement du cathéter et enfin le décès dans $6 \%$ des cas. 


\begin{tabular}{|c|c|c|}
\hline & $\begin{array}{c}\text { Repères } \\
\text { Anatomiques }\end{array}$ & Echoguidage \\
\hline Variables & $\mathbf{n}=\mathbf{9 0}$ & $\mathrm{n}=86$ \\
\hline Age (années), moyenne \pm DS & $61 \pm 16$ & $61 \pm 15$ \\
\hline Hommes, n (\%) & $52(58)$ & $54(63)$ \\
\hline Echelle de MacCabe (1/2/3), \% & $78 / 18 / 2$ & $71 / 20 / 10$ \\
\hline IGS II, moyenne \pm DS & $50 \pm 15$ & $50 \pm 15$ \\
\hline IMC $\left(\mathrm{kg} \cdot \mathrm{cm}^{-2}\right)$, moyenne \pm DS & $39 \pm 9$ & $37 \pm 5$ \\
\hline $\begin{array}{l}\text { Type d'admission, n (\%) } \\
\text { Médicale } \\
\text { Chirurgicale }\end{array}$ & $\begin{array}{l}55(61) \\
35(39)\end{array}$ & $\begin{array}{l}53(62) \\
33(38)\end{array}$ \\
\hline PAM, moyenne \pm DS & $77 \pm 18$ & $78 \pm 18$ \\
\hline $\mathrm{FC}$, moyenne $\pm \mathrm{DS}$ & $97 \pm 19$ & $95 \pm 22$ \\
\hline Ventilation Mécanique, n (\%) & $79(88)$ & $74(86)$ \\
\hline Catécholamines, n (\%) & $31(34,4)$ & $32(37,2)$ \\
\hline $\begin{array}{l}\text { Antibiothérapie, n (\%) } \\
\text { Aucune } \\
\text { Probabiliste } \\
\text { Adaptée }\end{array}$ & $\begin{array}{l}11(22) \\
21(42) \\
17(34)\end{array}$ & $\begin{array}{l}10(24) \\
21(51) \\
10(24)\end{array}$ \\
\hline $\begin{array}{l}\text { Anticoagulation, n (\%) } \\
\text { Aucune } \\
\text { Préventive } \\
\text { Curative }\end{array}$ & $\begin{array}{l}20(22) \\
45(50) \\
25(28)\end{array}$ & $\begin{array}{l}16(19) \\
44(51) \\
26(30)\end{array}$ \\
\hline $\begin{array}{l}\text { Leucocytes }\left(10^{3} \text { cellules. } \mathrm{mm}^{-3}\right) \\
\text { moyenne } \pm \text { DS }\end{array}$ & $19,1 \pm 31,3$ & $14,5 \pm 5,9$ \\
\hline $\begin{array}{l}\text { Plaquettes }\left(10^{3} \text { cellules. } \mathrm{mm}^{-3}\right) \\
\text { moyenne } \pm \mathrm{DS}\end{array}$ & $223,9 \pm 125,2$ & $260,1 \pm 145,7$ \\
\hline $\mathrm{TP}(\%)$, moyenne $\pm \mathrm{DS}$ & $66 \pm 17$ & $63 \pm 17$ \\
\hline TCA (s), moyenne \pm DS & $33 \pm 12$ & $35 \pm 16$ \\
\hline Durée de vie du cathéter $(\mathrm{j})$, moyenne \pm DS & $13 \pm 10^{*}$ & $11 \pm 10^{* *}$ \\
\hline
\end{tabular}

Les valeurs sont exprimées sous forme d'effectifs et de proportions ou moyenne \pm déviation standard (DS) $\mathrm{n}=$ nombre de patient $;{ }^{*}=$ analyse sur 67 patients $;{ }^{* *}=$ analyse sur 82 patients

IGS II = Indice de Gravité Simplifié II; IMC = Indice de Masse Corporelle ; PAM : pression artérielle moyenne ; FC : fréquence cardiaque ; TP = Temps de Prothrombine $; \mathrm{TCA}=$ Temps de Céphaline Activé. 


\section{Critères de jugement principal et secondaires}

Les résultats obtenus sont présentés dans le tableau 2.

Lorsque l'on s'intéresse au critère de jugement principal, nous avons trouvé un résultat statistiquement significatif en faveur du groupe « échoguidage », avec une réduction de $70 \%$ à $44 \%(p=0,0005)$ du taux de complications.

Pour les critères de jugement secondaires, la pose sous échoguidage a permis de réduire le taux de complications mécaniques de manière significative de $47 \%$ à $15 \%$ $(\mathrm{p}<0,0001)$, dont le taux d'échecs, qui apparaît comme étant inférieur dans le groupe « échoguidage » $(12 \%$ vs $44 \% ; \mathrm{p}<0,0001)$. En revanche, on ne peut conclure en ce qui concerne les taux d'hématome, de ponction artérielle et de pneumothorax. Dans le même sens, le temps de pose apparaît significativement réduit $(6,4 \min \pm 6,3$ vs $3,9 \pm 4,2$; $\mathrm{p}=0,0025)$ ainsi que le nombre de ponctions réalisées sous échoguidage $(2,2 \pm 1,4$ vs $1,5 \pm 0,9$; $\mathrm{p}<0,0001)$.

Pour les complications infectieuses, il n'y a pas de différence significative retrouvée entre les deux bras.

Nous avons au total comptabilisé 34 colonisations de CVC, ce qui représente $27 \%$ de l'ensemble des cathéters mis en culture. Les germes les plus fréquemment en cause sont le groupe représenté par les bacilles gram négatifs (53\%) puis la famille des staphylocoques à coagulase négative $(20 \%)$, les levures $(12 \%)$, le staphylocoque doré $(9 \%)$ et enfin les entérocoques $(6 \%)$.

Parmi les bacilles gram négatifs, nous avons relevé 38\% de CVC colonisés à Pseudomonas aeruginosa, 16\% à Serratia marcescens, 11\% à Enterobacter cloacae, 11\% à Stenotrophomonas maltophilia et enfin, E.Coli, Acinetobacter baumanii, Enterobacter aerogenes et K.pneumoniae avec respectivement $6 \%$ chacune. Sur l'ensemble des cathéters, aucune infection liée au cathéter n'a été recensée. Nous avons effectivement retrouvé deux hémocultures positives, mais les germes identifiés étaient différents de ceux responsables de la colonisation du cathéter.

Sur les 126 patients chez qui nous avons réalisé un écho doppler de contrôle dans les quatre jours suivants l'ablation du cathéter, 17 patients (13\%) ont présenté une complication thrombotique. Pour ce type de complication, nous n'avons pas montré de différence significative entre les deux groupes étudiés. 
En effet, on passe de $20 \%$ de complications dans le bras « repères anatomiques » à seulement $9 \%$ dans le bras « échoguidage » avec un $\mathrm{p}=0,08$.

Tableau 2 : Critères de jugement principal et secondaires

\begin{tabular}{|c|c|c|c|}
\hline & Repères & Echoguidage & \\
\hline & $\mathbf{n}=\mathbf{9 0}$ & $\mathrm{n}=86$ & $p$ \\
\hline \multicolumn{4}{|l|}{ Critère de jugement principal } \\
\hline Complications (critère composite), n (\%) & $63(70)$ & $38(44)$ & 0,0005 \\
\hline \multicolumn{4}{|l|}{ Critères de jugements secondaires } \\
\hline Complications Mécaniques, n (\%) & $42(47)$ & $13(15)$ & $<0,0001$ \\
\hline Echec, n (\%) & $40(44)$ & $10(12)$ & $<0,0001$ \\
\hline Hématome, n (\%) & $4(4)$ & $3(3)$ & $>0,9$ \\
\hline Ponction artérielle, n (\%) & $6(7)$ & $2(2)$ & 0,30 \\
\hline Pneumothorax, n (\%) & 0 & 0 & \\
\hline Complications Infectieuses, $\mathrm{n} / \mathrm{n}$ total (\%) & $15 / 50(30)$ & $19 / 76(25)$ & 0,53 \\
\hline Complications Thrombotiques, $\mathrm{n} / \mathrm{n}$ total (\%) & $10 / 50(20)$ & $7 / 76(9)$ & 0,08 \\
\hline Temps de pose $(\mathrm{mn})$, moyenne $\pm \mathrm{DS}$ & $6,4 \pm 6,3$ & $3,9 \pm 4,2$ & 0,0025 \\
\hline Infection liée au cathéter, n (\%) & 0 & 0 & \\
\hline Nombre de ponctions, moyenne \pm DS & $2,2 \pm 1,4$ & $1,5 \pm 0,9$ & $<0,0001$ \\
\hline Mortalité en réanimation, n (\%) & $17(19)$ & $13(15)$ & 0,50 \\
\hline
\end{tabular}

Les valeurs sont exprimées sous forme d'effectifs et de proportions ou moyenne \pm déviation standard (DS) $\mathrm{n}=$ nombre de patient $; \mathrm{mn}=$ minute 
La figure 2 présente les critères de jugement principal et secondaires à l'aide d'un diagramme.

\section{Figure 2 Critères de jugement principal et secondaires}

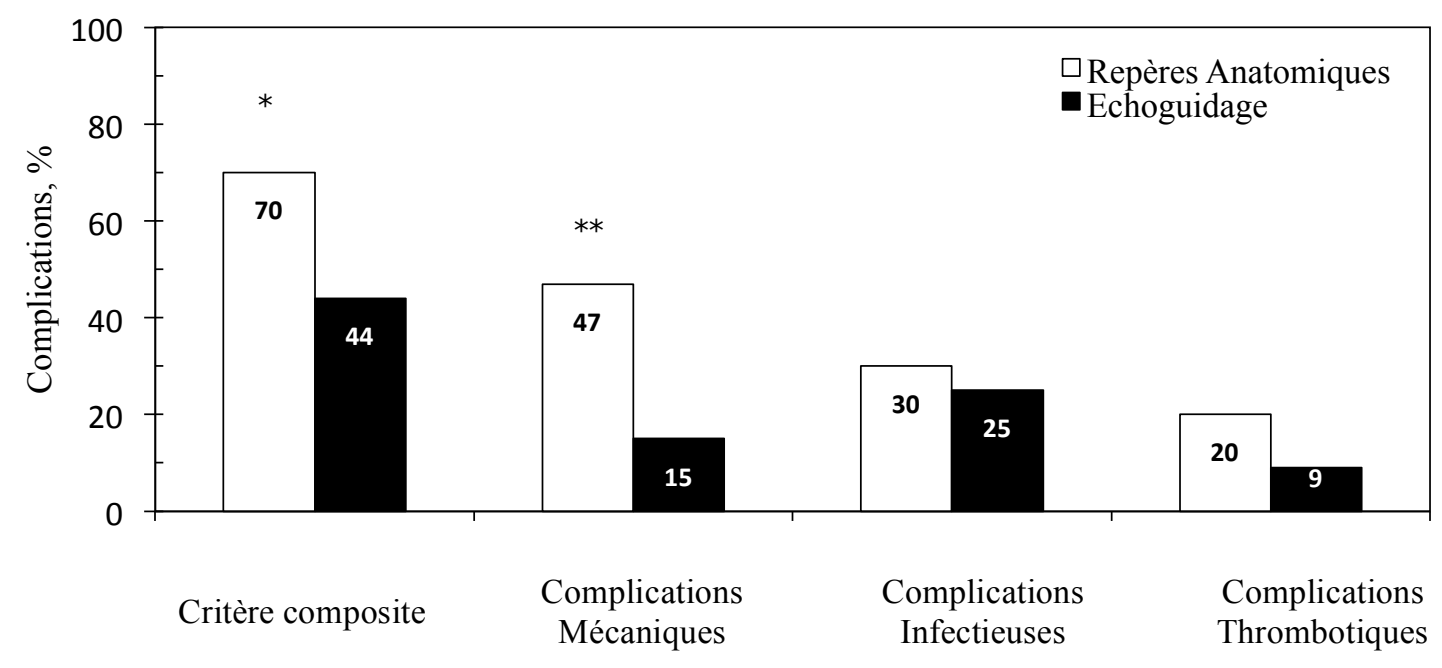

${ }_{\mathrm{p}}^{*}=0,0005$ vs groupe « échoguidage $»,{ }^{* *} \mathrm{p}<0,0001$ vs groupe échoguidage

\section{Analyse en sous-groupe selon l'IMC}

Nous avons ensuite étudié ces mêmes critères de jugement principal et secondaires dans 3 sous-groupes différents en fonction de la sévérité de l'obésité:

obésité modérée $\left(30 \leq \mathrm{IMC} \leq 34,9 \mathrm{~kg} / \mathrm{m}^{2}\right)$, obésité sévère $\left(35 \leq \mathrm{IMC} \leq 39,9 \mathrm{~kg} / \mathrm{m}^{2}\right)$ et enfin obésité morbide (IMC $\geq 40 \mathrm{~kg} / \mathrm{m}^{2}$ ).

Les principaux résultats sont présentés dans le tableau 3.

Pour le critère de jugement principal, nous avons retrouvé une différence significative en faveur du groupe "échoguidage » seulement pour le sous-groupe des patients présentant une obésité modérée $(p=0,0005)$.

Pour les complications mécaniques, l'utilisation de l'échoguidage entraîne une réduction de ce type de complications, avec des résultats qui sont statistiquement significatifs dans les 3 sous-groupes $(p=0,0045, p=0,02$ et $p=0,009$ respectivement pour les sousgroupes de patients avec une obésité modérée, sévère et morbide).

De la même façon, la proportion des échecs de pose est réduite par l'utilisation de l'échoguidage dans les 3 sous-groupes, de $60 \%, 50 \%$ et $67 \%$ respectivement pour les patients avec une obésité modérée, sévère et morbide $(p=0,0013, p=0,002$ et $p=0,006)$. 


\begin{tabular}{|c|c|c|c|}
\hline & $\begin{array}{c}\text { Repères } \\
\text { anatomiques }\end{array}$ & Echoguidage & $\mathbf{p}$ \\
\hline \multicolumn{4}{|l|}{ Critère de jugement principal } \\
\hline Complications (Critère composite), $\mathrm{n} / \mathrm{n}$ total (\%) & & & 0,0005 \\
\hline $\operatorname{IMC}(30-34,9)$ & $25 / 33(76)$ & $11 / 33(33)$ & 0,0005 \\
\hline $\operatorname{IMC}(35-39,9)$ & $15 / 23(65)$ & $14 / 27(50)$ & 0,34 \\
\hline $\mathrm{IMC} \geq 40$ & $23 / 34(68)$ & $13 / 26(50)$ & 0,16 \\
\hline \multicolumn{4}{|l|}{ Critères de jugements secondaires } \\
\hline Complications Mécaniques, $\mathrm{n} / \mathrm{n}$ total (\%) & & & $<0,0001$ \\
\hline $\operatorname{IMC}(30-34,9)$ & $17 / 33(52)$ & $6 / 33(18)$ & 0,0045 \\
\hline $\operatorname{IMC}(35-39,9)$ & $9 / 23(39)$ & $3 / 27(11)$ & 0,02 \\
\hline $\mathrm{IMC} \geq 40$ & $16 / 34(47)$ & $4 / 26(15)$ & 0,009 \\
\hline Complications Infectieuses, $\mathrm{n} / \mathrm{n}$ total $(\%)$ & & & 0,53 \\
\hline $\operatorname{IMC}(30-34,9)$ & $5 / 17(29)$ & $4 / 29(14)$ & 0,36 \\
\hline $\operatorname{IMC}(35-39,9)$ & $3 / 14(21)$ & $8 / 25(32)$ & 0,73 \\
\hline $\mathrm{IMC} \geq 40$ & $7 / 19(37)$ & $7 / 23(30)$ & 0,66 \\
\hline Complications Thrombotiques, $\mathrm{n} / \mathrm{n}$ total (\%) & & & 0,08 \\
\hline $\operatorname{IMC}(30-34,9)$ & $4 / 17(24)$ & $1 / 29(3)$ & 0,055 \\
\hline $\operatorname{IMC}(35-39,9)$ & $3 / 14(21)$ & $3 / 25(12)$ & 0,74 \\
\hline $\mathrm{IMC} \geq 40$ & $3 / 19(16)$ & $3 / 23(13)$ & $>0,9$ \\
\hline Temps de pose $(\mathrm{mn})$, moyenne \pm DS & & & 0,0025 \\
\hline $\operatorname{IMC}(30-34,9)$ & $6,9 \pm 7$ & $3,8 \pm 3,2$ & 0,02 \\
\hline $\operatorname{IMC}(35-39,9)$ & $5,4 \pm 4,2$ & $3,8 \pm 4,6$ & 0,20 \\
\hline $\mathrm{IMC} \geq 40$ & $6,6 \pm 6,7$ & $4,4 \pm 4,8$ & 0,15 \\
\hline Nombres de ponction, moyenne \pm DS & & & $<0,0001$ \\
\hline $\operatorname{IMC}(30-34,9)$ & $2,4 \pm 1,8$ & $1,5 \pm 0,9$ & 0,01 \\
\hline $\operatorname{IMC}(35-39,9)$ & $2 \pm 1,2$ & $1,4 \pm 0,9$ & 0,04 \\
\hline $\mathrm{IMC} \geq 40$ & $2,1 \pm 1$ & $1,5 \pm 0,8$ & 0,008 \\
\hline
\end{tabular}

Les valeurs sont exprimées sous forme d'effectifs et de proportions ou moyenne \pm déviation standard (DS) $\mathrm{n}=$ nombre de patient $; \mathrm{mn}=$ minute $; \mathrm{IMC}=$ Indice de Masse Corporelle. 
Le temps de pose est plus court grâce à l'échoguidage chez le sous-groupe de patients avec une obésité modérée $(\mathrm{p}=0,02)$ exclusivement.

Enfin on réalise moins de ponctions lors de la pose des CVC par technique échoguidée chez les patients obèses, quelle que soit leur IMC $(p=0,01, p=0,04$ et $p=0,008$ respectivement pour le sous-groupe de patients avec une obésité modérée, sévère et morbide).

En revanche, nous n'avons pas retrouvé de différence significative, en terme de critère de jugement principal, entre les différents sous-groupes d'IMC, ni pour les complications infectieuses et thrombotiques.

\section{Analyse en sous-groupe selon de l'expérience de l'opérateur}

Nous avons finalement étudié ces mêmes critères de jugement principal et secondaires dans 3 sous-groupes différents en fonction de l'expérience de l'opérateur : niveau A (internes de $1^{\text {ère }}$ et $2^{\text {ème }}$ année), niveau $\mathrm{B}$ (internes de $3^{\text {ème }}$ et $4^{\text {ème }}$ année) et niveau $\mathrm{C}$ (internes de $5^{\text {ème }}$ année et séniors).

Les principaux résultats sont présentés dans le tableau 4.

Pour le critère de jugement principal, il apparaît une significativité statistique en faveur de l'échoguidage dans le sous-groupe de niveau B $(\mathrm{p}=0,005)$. Aucune différence n'apparaît dans les autres sous-groupes.

Pour les complications mécaniques, celles-ci sont statistiquement inférieures grâce à l'échoguidage dans le même sous-groupe de niveau B $(p<0,0001)$. Ainsi, dans le sousgroupe de niveau $\mathrm{B}$, l'échoguidage a permis de réduire le taux d'échec de $52 \%$ à $9 \%$ $(p<0,0001)$, le temps de pose $(p=0,0032)$ et le nombre de ponctions $(p<0,0001)$.

En revanche, en ce qui concerne les complications infectieuses et thrombotiques, aucune différence statistiquement significative n'a été mise en évidence dans aucun des trois sous-groupes.

Enfin, nous n'avons relevé aucune différence significative, entre les différents sousgroupes de niveau d'expérience, en terme de survenue du critère de jugement principal. 


\begin{tabular}{|c|c|c|c|}
\hline & $\begin{array}{c}\text { Repères } \\
\text { anatomiques }\end{array}$ & Echoguidage & $\mathbf{p}$ \\
\hline \multicolumn{4}{|l|}{ Critère de jugement principal } \\
\hline Complications (Critère composite), $\mathrm{n} / \mathrm{n}$ total (\%) & & & 0,0005 \\
\hline Niveau A & $5 / 6(83)$ & $5 / 9(56)$ & 0,55 \\
\hline Niveau B & $45 / 61(74)$ & $20 / 43(47)$ & 0,005 \\
\hline Niveau C & $13 / 23(57)$ & $13 / 34(38)$ & 0,17 \\
\hline \multicolumn{4}{|l|}{ Critères de jugements secondaires } \\
\hline Complications Mécaniques, $\mathrm{n} / \mathrm{n}$ total (\%) & & & $<0,0001$ \\
\hline Niveau A & $1 / 6(17)$ & $3 / 9(33)$ & 0,6 \\
\hline Niveau B & $33 / 61(54)$ & $5 / 43(12)$ & $<0,0001$ \\
\hline Niveau C & $8 / 23(35)$ & $5 / 34(15)$ & 0,07 \\
\hline Complications Infectieuses, $\mathrm{n} / \mathrm{n}$ total $(\%)$ & & & 0,53 \\
\hline Niveau A & $4 / 5(80)$ & $1 / 6(17)$ & 0,12 \\
\hline Niveau B & $9 / 29(31)$ & $12 / 39(31)$ & 0,98 \\
\hline Niveau C & $2 / 16(13)$ & $6 / 31(19)$ & 0,84 \\
\hline Complications Thrombotiques, $\mathrm{n} / \mathrm{n}$ total (\%) & & & 0,08 \\
\hline Niveau A & $0 / 5$ & $1 / 6(17)$ & $>0,9$ \\
\hline Niveau B & $7 / 29(24)$ & $4 / 39(10)$ & 0,22 \\
\hline Niveau C & $3 / 16(19)$ & $2 / 31(6)$ & 0,32 \\
\hline Temps de pose (mn), moyenne \pm DS & & & 0,0025 \\
\hline Niveau A & $5,3 \pm 4,8$ & $8,7 \pm 6,5$ & 0,30 \\
\hline Niveau B & $7,5 \pm 6,9$ & $3,8 \pm 4,2$ & 0,0032 \\
\hline Niveau C & $3,9 \pm 3,5$ & $2,8 \pm 2$ & 0,12 \\
\hline Nombres de ponction, moyenne \pm DS & & & $<0,0001$ \\
\hline Niveau A & $1,5 \pm 0,8$ & $2,4 \pm 1,1$ & 0,10 \\
\hline Niveau B & $2,4 \pm 1,5$ & $1,3 \pm 0,7$ & $<0,0001$ \\
\hline Niveau C & $1,8 \pm 0,9$ & $1,4 \pm 0,9$ & 0,12 \\
\hline
\end{tabular}

Les valeurs sont exprimées sous forme d'effectifs et de proportions ou moyenne \pm déviation standard (DS) $\mathrm{n}=$ nombre de patient $; \mathrm{mn}=$ minute $;$ Niveau $\mathrm{A}=$ Internes $1^{\text {ere }}$ année $+2^{\text {ème }}$ année $;$ Niveau $\mathrm{B}=$ Internes $3^{\text {ème }}$ année $+4^{\text {ème }}$ année $;$ Niveau $\mathrm{C}=$ Internes $5^{\text {ème }}$ année + Séniors 


\section{DISCUSSION}

Il s'agit du premier travail évaluant le bénéfice de l'échoguidage pour la pose des cathéters veineux centraux par voie jugulaire interne chez les patients obèses en réanimation. Pendant toute la durée de l'étude, 176 patients ont été inclus, 90 dans le groupe « repères anatomiques » et 86 dans le groupe « échoguidage ». Les caractéristiques des patients étaient identiques entre les deux groupes. Le taux de complications, correspondant au critère de jugement principal, était significativement inférieur dans le groupe «échoguidage » (44\%) par rapport au groupe « repères anatomiques» $(70 \%)$.

\section{Intérêt de l'échoguidage}

Notre étude montre que la technique de pose des CVC par échoguidage devrait être la technique de choix. De nombreuses études, analysant les données de patients tous poids confondus, ont aussi établi la supériorité de cette technique [14, 15, 18, 64-69], ainsi qu'au moins trois méta-analyses [10, 35, 67]. Dans ces travaux, la technique d'échoguidage augmente le taux de succès de pose des CVC et décroît considérablement le taux de complications. Cela a conduit les sociétés savantes à recommander l'utilisation de cette technique dans la pose des CVC [24-27].

Denys et coll. sont les premiers à s'être intéressés, dans une étude à grande échelle, à l'apport de l'échoguidage avec un système de porte-aiguille semblable à celui que nous avons utilisé. Ce système permettait de diminuer le taux d'échec, le temps de pose et le taux de complications comparativement au repérage anatomique [69].

Dans la grande majorité des études sur l'échoguidage, la sonde d'échographie est positionnée de telle sorte que les vaisseaux sont visualisés selon une coupe «petit axe ». Il faut savoir que la technique échoguidée entraîne dans 34\% des cas une transfixion du mur postérieur de la veine ponctionnée [70], avec un risque d'atteinte des structures voisines. Stone et coll. dans leur étude sur des mannequins, montrent que la coupe « long axe » permet une meilleure visualisation de l'extrémité de l'aiguille et ainsi une diminution des transfixions du mur postérieur de la veine et des complications associées [71]. 
En revanche, dans une étude portant sur 99 patients de chirurgie cardiaque nécessitant la pose préopératoire d'un $\mathrm{CVC}$ en jugulaire interne, la coupe "petit axe » permettait d'accroître le nombre de réussite de pose du CVC du premier coup et nécessitait moins de changements de direction de l'aiguille de ponction [72].

Dans notre étude, le système du porte-aiguille nous imposait, avec l'échographe dédié, de se positionner selon une coupe « petit axe ».

\section{Critères de jugement principal et secondaires}

a. Critère de jugement principal

Dans notre travail, nous avons choisi comme critère de jugement principal un critère composite, qui englobe les trois types de complications que nous avons décrits. En effet, la survenue d'une complication mécanique et/ou infectieuse et/ou thrombotique, rendait l'événement « complication » comme présent.

La plupart des études sur la comparaison des techniques ou sites de pose des cathéters veineux centraux mettent en avant, comme critère de jugement principal, le succès de cannulation du cathéter $[16,23]$, ou alors un seul type de complication, le taux de complications infectieuses dans l'étude de Lorent et coll. [73], ou le taux de complications mécaniques dans celle de Schummer et coll. [36].

A notre connaissance, il s'agit du premier travail qui fixe comme critère de jugement principal la survenue d'au moins un des trois types de complications engendrées par les cathéters veineux centraux. Cela nous donne la particularité de pouvoir comparer les deux techniques de pose des CVC étudiées, avec la morbidité globale que chacune d'entre elles engendre.

\section{b. Critères de jugement secondaires}

Dans notre travail, nous avons relevé un taux de complications mécaniques significativement inférieur dans le groupe «échoguidage» comparativement au groupe « repérage anatomique ». Celles-ci comprennent l'échec de pose, l'hématome et la ponction artérielle. Nous n'avons pas relevé de pneumothorax, ni d'hémothorax. 
Il est intéressant de noter que le taux de complications de $47 \%$ dans le groupe « repères anatomiques » est deux fois supérieur à celui de la population globale, tous poids confondus, technique et site de pose identiques [16,74], d'où l'intérêt que nous avons porté à cette population spécifique représentée par les patients obèses, pour laquelle le bénéfice apporté par l'échoguidage aurait un impact considérable.

Les données de la littérature corroborent ces résultats. Airapetian et coll. [16] ont comparé trois techniques de pose (échoguidage, échorepérage et repérage anatomique). L'échoguidage permettait une réduction du taux de complications mécaniques de $25 \%$ dans le sous-groupe en jugulaire interne. Une méta-analyse récente de 2013 comparant nos deux mêmes techniques de pose de $\mathrm{CVC}$, confirme nos résultats en concluant que l'échoguidage permet une réduction des complications mécaniques en réduisant le risque d'échec, de ponction artérielle, d'hématome, de pneumothorax et d'hémothorax chez les patients adultes [35].

Lorsque l'on s'intéresse plus spécifiquement au taux d'échec, nous avons démontré que l'échoguidage permettait de réduire significativement cette complication précoce, de 44\% à $12 \%$. Dans l'étude d'Airapetian et coll. l'échec était de $25 \%$ en repérage anatomique, et nul en échoguidage [16]. Dans celle de Karakitsos et coll., qui compare les deux techniques de poses en jugulaire interne chez 900 patients de réanimation, l'échec était de seulement 5,6\% en repérage anatomique et nul à nouveau en échoguidage [14]. Enfin, dans l'étude de Schummer et coll., qui s'intéresse aux complications mécaniques des CVC posés en repérage anatomique, dans le sous-groupe de ceux posés en jugulaire interne, le taux d'échecs était seulement de 5,9\% [36].

Dans la littérature, les taux d'échecs de pose sont donc inférieurs. Nous pensons que cela est expliqué par le fait que les populations sont différentes, l'IMC moyen des études variant entre 24 et $26 \mathrm{Kg} / \mathrm{m}^{2}$, l'obésité serait certainement un facteur de risque surajouté d'échec de pose. De plus la définition du succès de pose dans la littérature est beaucoup plus large que celle de notre étude où un nombre de ponctions $<3$ était nécessaire.

En ce qui concerne les autres complications mécaniques comme la ponction artérielle et l'hématome, nous n'avons pas montré de différence significative entre les deux groupes étudiés. Nous n'avons pas relevé de pneumothorax, dans aucun des deux bras. Notre taux de ponctions artérielles dans le groupe « repérage anatomique» $(7 \%)$ est une nouvelle fois plus élevé que celui retrouvé dans l'étude de Schummer et coll. dans une population tous poids confondus $(4,6 \%)[36]$. 
L'incidence des complications mécaniques est 6 fois plus élevée lorsque le même opérateur effectue plus de 3 tentatives de ponctions [34]. L'étude de Schummer et coll. a démontré qu'un nombre de ponctions cutanées à la pose du $\mathrm{CVC} \geq 2$ était un facteur de risque d'échec de pose et des autres complications mécaniques [36].

Dans l'étude de Mansfield et coll., les poses de CVC qui nécessitaient plus de 2 ponctions cutanées avaient un taux d'échec de $43 \%$ et de complications mécaniques de $24 \%$ [75]. L'étude d'Eisen et coll. corrobore ces résultats [76]. Dans notre travail, nous avons montré que poser un CVC chez un patient obèse en jugulaire interne par échoguidage permet de diminuer significativement le nombre de ces ponctions cutanées en dessous de ce seuil de 2 ponctions.

Dans notre travail, nous n'avons pas retrouvé de différence significative pour les complications infectieuses entre les deux techniques de pose. Leur taux dans notre population globale est de $27 \%$ parmi les CVC cultivés, et correspond uniquement à des colonisations de cathéters (résultat de la culture au dessus du seuil de positivité à $10^{3}$ UFC/ml). Nous n'avons relevé aucune bactériémie, ni aucune infection liée au cathéter.

Rares sont les études qui se sont intéressées à l'apport de l'échographie dans ce domaine. L'étude de Karakitsos et coll. rapporte un taux de septicémie lié au CVC diminué dans le groupe échoguidé [14].

Le taux de colonisations que nous avons recensé dans notre travail est plus élevé par rapport aux autres études sur le sujet. Dans RéaRaisin 2012, ils comptent 12,1\% de colonisations parmi les CVC mis en culture [77], alors que dans l'étude de Lorente et coll. un taux très inférieur à 4,43\%, qui peut être expliqué par leur définition plus stricte associant à la colonisation un signe local d'infection (signes inflammatoires cutanés, purulence..) [73]. Dans l'étude Cathédia, le taux de colonisation est de $25 \%$, mais il s'agit de cathéters de dialyse [42].

Tous ces résultats suggèrent que le risque de colonisation des CVC chez l'obèse est plus élevé que chez les patients non obèses, et que ce risque ne diminue pas avec l'échoguidage.

Lorsque l'on s'intéresse aux germes retrouvés, les bacilles à gram négatif sont largement prépondérants (53\%) par rapport aux bactéries à gram positif (35\%). Ces proportions sont inversées ou égales dans la plupart des études [42, 77]. 
Le taux de complications thrombotiques dans notre travail est de $13 \%$ dans la population globale. Dans la littérature, l'incidence de cette complication est comprise entre $2 \%$ et $26 \%$, nos résultats sont donc concordants [14, 39, 43, 78].

Nous n'avons pas retrouvé de différence significative entre nos deux groupes de randomisation, même si le résultat pourrait être en faveur de l'échoguidage avec plus de patients $(\mathrm{p}=0.08)$.

En plus, l'échoguidage permet aussi de vérifier la perméabilité des veines à ponctionner et détecter les malpositions vasculaires.

Enfin, lorsque l'on s'attarde sur le temps de pose, nos résultats nous montrent que la pose d'un CVC est plus rapide grâce à l'échoguidage dans notre population de patients obèses.

Il n'existe pas de réelles données objectives qui prouvent que plus la pose du CVC est rapide, moins elle engendre de complications. Il semble tout de même judicieux de penser que moins la durée d'insertion d'un CVC est longue, moins on s'expose à des risques notamment infectieux (matériel moins exposé, moins de manipulations, moins de souillures sanguines...). Par ailleurs, un temps de pose long est généralement lié à des difficultés de pose et donc à une multiplication du nombre de ponctions cutanées, or nous avons déjà établi le lien entre un nombre de ponctions cutanées élevé et l'augmentation des complications mécaniques. Cela est aussi vrai avec les complications infectieuses, en augmentant le risque de bactériémies liées au CVC [14]. Dans la littérature, de nombreuses études ont démontré que l'utilisation de l'échoguidage permettait de réduire le temps de pose et le temps de ponction veineuse $[10,14$, $16,23,67]$.

Donc, au delà de la satisfaction personnelle bien trop souvent mise en avant, la pose « rapide » d'un cathéter représente un réel enjeux en termes de diminution des complications, et pour cela la technique d'échoguidage est la technique de choix. 


\section{Analyse en sous-groupe}

a. Selon l'IMC des patients

Aux Etats-Unis, la prévalence de l'obésité est passée de 23\% en 1998-1994, à 30\% en 1999-2000 [79]. Au Royaume-Uni, elle a doublé entre 1980 et 1991, passant de 6\% à 13\% chez l'homme, et de $8 \%$ à $15 \%$ chez la femme. En France, l'obésité concernait 15\% des adultes en 2012, contre seulement 6,1\% en 1980 [80]. La prévalence des patients obèses en réanimation médicochirurgicale est estimée entre $9 \%$ et $26 \%$ [52].

La première étude s'étant intéressée spécifiquement au pronostic des sujets obèses en réanimation médicale a été publiée en 2001 [81]. Depuis d'autres études on été publiées avec des conclusions contradictoires quant à l'augmentation de la mortalité dans cette population spécifique. Seule une étude prospective retrouve une augmentation de la mortalité de manière significative chez les patients obèses par rapport aux reste des patients de réanimation $(32 \%$ vs $13 \%$ avec $\mathrm{p}<0,001)$. En analyse multivariée, un IMC supérieur à $27 \mathrm{Kg} / \mathrm{m}^{2}$ était identifié comme étant un facteur indépendant de mortalité [82].

Dans notre étude, nous avons réparti les patients inclus en trois sous-groupes en fonction de la sévérité de leur obésité : moyenne, sévère et morbide. Par ailleurs, le sousgroupe d'IMC qui semble tirer le plus de bénéfices par l'utilisation de l'échoguidage est celui du niveau d'obésité modéré, avec un IMC compris entre $30 \mathrm{Kg} / \mathrm{m}^{2}$ et $34,9 \mathrm{Kg} / \mathrm{m}^{2}$.

Après analyse des données, il semble que seuls les patients avec une obésité moyenne bénéficient pleinement de l'échoguidage en réduisant de moitié le taux de complications, le nombre de ponctions et le temps de pose. L'échoguidage pourrait également réduire la proportion des thromboses sur cathéters. Par contre, les patients avec une obésité sévère et morbide tirent bénéfice de l'échoguidage en baissant le taux de complications mécaniques et le nombre de ponctions.

A ce jour, il est difficile de comparer aux autres études car, soit elles ne s'intéressaient pas à la population d'obèses [14], soit elles ne précisaient pas l'IMC des patients [23]. 


\section{b. Selon l'expérience de l'opérateur}

Dans notre travail, nous avons réparti les patients inclus en trois sous-groupes, selon l'expérience de l'opérateur : niveau A (internes $1^{\text {ère }}$ et $2^{\text {ème }}$ année), niveau B (internes de $3^{\text {ème }}$ et $4^{\text {ème }}$ année) et enfin niveau $\mathrm{C}$ (internes 5ème année et séniors). Nos résultats montrent que l'échoguidage apporte un bénéfice aux opérateurs de niveau $B$, avec une réduction significative du critère de jugement principal, des complications mécaniques, des échecs de pose, du temps de pose et enfin du nombre de ponctions. Ceci pourrait s'expliquer par le fait qu'à ce stade de la formation, l'expérience en situation de repérage anatomique ne soit pas aussi élevée que pour les séniors, mais que l'utilisation de l'outil échographique soit au contraire parfaitement maîtrisé. Dans le sous-groupe de niveau C, l'échoguidage aurait tendance, avec des résultats proches de la significativité, à réduire les complications mécaniques $(p=0,07)$ et le taux d'échec $(p=0,08)$. Probablement avec un effectif supérieur nous aurions pu obtenir des résultats significatifs. Dans le sous-groupe de niveau A représentant les internes plus inexpérimentés, il n'existe aucune différence entre les deux techniques de pose. Il faut noter que l'effectif des internes dans ce sous-groupe est particulièrement faible, engendrant un manque de puissance indiscutable.

Les données de la littérature sont contradictoires concernant l'influence de l'expérience de l'opérateur sur les taux de complications lors de la pose d'un CVC.

Certaines études montrent que l'inexpérience est pourvoyeuse de complications, et qu'il y a une différence significative en faveur d'un opérateur expérimenté pour obtenir moins de complications mécaniques dans leur ensemble [76], et tout particulièrement moins de ponctions artérielles [83].

D'autres soulignent des taux de succès et de complications proches entre les jeunes opérateurs et ceux plus expérimentés. Plusieurs études, dès les années 1990, montrent que le taux de succès avec l'échoguidage est très bon même avec une faible expérience [16, 84], d'autres, comme celle de Schummer et coll., trouvent que même un opérateur expérimenté peut être à l'origine d'un nombre considérable de complications mécaniques et de malpositions [36].

Dans tous les cas, lorsque que l'on s'intéresse à la technique de pose utilisant l'échoguidage ou le repérage anatomique, les données de la littérature nous montrent que, même chez des opérateurs inexpérimentés, l'échoguidage apporte un bénéfice lors de la pose d'un CVC, permettant une diminution des complications mécaniques, un nombre de ponctions inférieur et un temps de pose plus rapide [16]. 
On pourrait ainsi penser que l'échoguidage permet de gommer l'inexpérience, et nous offre une ponction plus sûre quelle que soit l'expérience de la personne qui ponctionne. Par conséquent il paraît judicieux de proposer initialement aux médecins en formation un apprentissage de la pose d'un CVC par la technique échoguidée, afin d'éviter les complications liées à l'inexpérience. L'apprentissage de la technique de pose par simple repérage anatomique interviendrait dans un deuxième temps, car il est admis que les médecins doivent rester capables de maîtriser cette technique, notamment pour les situations d'urgence ou encore lorsque l'appareil d'échographie n'est pas disponible.

Certains se sont intéressés à l'apport d'un enseignement par simulateur (mannequin sur lequel il était possible d'effectuer l'échoguidage et la ponction veineuse), qui a démontré que ce type d'apprentissage permettait de réduire significativement le taux de complications, spécialement chez les opérateurs inexpérimentés [85]. Cependant le coût d'un tel appareil est inévitablement élevé et difficile à mettre en place à grande échelle, alors que finalement la courbe d'apprentissage de la pose d'un CVC sous échoguidage est plutôt aisée et accessible, car en jugulaire interne seulement 4 à 8 insertions seraient nécessaires afin d'en acquérir la compétence [32].

\section{Evolution des recommandations}

A l'étranger, les recommandations concernant la mise en place des cathéters veineux centraux existent depuis de nombreuses années.

Aux Etats-Unis, dès 2001, l'échoguidage est recommandé pour diminuer les complications, et fait partie des 11 recommandations pratiques s'intéressant à la sécurité des patients de la « Agency for Healthcare Research and Quality » [86].

En 2002, au Royaume-Unis, le National Institute of Clinical Excellence (NICE) publie des recommandations sur la pose des CVC en jugulaire interne et précise que l'échoguidage est la technique à utiliser. Les professionnels concernés doivent en recevoir une formation appropriée.

En 2011, la «American Society of Echocardiography and Cardiovascular Anesthesiologists » statuent que la technique d'échoguidage doit être utilisée chaque fois que possible pour la pose en position jugulaire interne, pour minimiser les échecs et les autres complications [25]. 
En 2012, l'« American Society of Anesthesiologists » elle aussi, pour la pose des CVC en jugulaire interne, préconise l'utilisation de l'échoguidage chaque fois que possible, et même qu'un échorepérage doit être effectué avant la pose des champs stériles, pour repérer les position des vaisseaux et confirmer leur perméabilité [26].

En France il aura fallu attendre 2014 avant que la SFAR n'établisse des Recommandations Formalisées d'Experts à ce sujet, stipulant que l'utilisation de l'échoguidage, par rapport à la technique de repérage anatomique, est la technique de choix (GRADE 1+) pour les CVC en jugulaire interne [33].

Mais avec toutes ces recommandations françaises et internationales, qu'en est-il de leur application en pratique? Deux enquêtes réalisées auprès de médecins anesthésistes anglais et canadiens montrent une utilisation encore faible de l'échographie [29, 87]. Entre $40 \%$ et $70 \%$ des médecins déclarent ne jamais avoir recours à l'appareil d'échographie pour la pose d'un CVC.

La première hypothèse pour expliquer cette réticence pourrait être un simple problème d'accès à l'appareil d'échographie lié au coût ou à l'absence de formation. Mais dans son analyse de coût, Calvert et coll. en 2004 ont montré, grâce à l'utilisation de l'échoguidage, la possibilité de réaliser une économie de $2000 £$ par 1000 procédures et 90 complications évitées [88]. Deuxièmement, avoir recours à l'appareil d'échographie pour la pose d'un CVC est trop souvent perçu comme une démonstration d'un manque de performance technique. Ces médecins qui sont réticents à l'utilisation de l'échoguidage, craignent, par ailleurs, que la dextérité de la pose des CVC «en aveugle» ne soit perdue, alors que cette technique doit rester maîtrisée en cas de non accès à l'échographe et dans les situations d'urgence.

En toute logique il est de notre devoir, surtout depuis les RFE de la SFAR de Décembre 2014 de privilégier encore plus cette technique d'insertion. Surtout au sein d'une population de patients obèses caractérisée comme plus à risque de complications, et ce d'autant plus que l'échoguidage permettrait de réaliser des économies. 


\section{Limites de l'étude}

Cette étude présente certaines limites à prendre en considération :

- l'effectif déterminé de départ (346 patients) n'a pas été atteint et nous avons arrêté notre travail à l'analyse intermédiaire à la moitié des effectifs. Il existe probablement un manque de puissance, notamment pour l'analyse des critères de jugement secondaires et l'analyse en sous-groupe.

- l'obésité était un des critères d'inclusion. Or nous avons décidé d'inclure les patients dont l'IMC le jour de la pose du CVC était supérieur à $30 \mathrm{Kg} / \mathrm{m}^{2}$. Nous sommes conscients que ce chiffre reflète un poids global sans tenir compte ni du type ni de la répartition de la masse du patient. De plus, nous n'avons pas mesuré le tour de cou, qui aurait pu être un critère de jugement secondaire à analyser.

- l'appareil utilisé est un appareil ad hoc, qui n'est, à l'heure actuelle, pas disponible dans tous les services de réanimation. Nous pouvons nous poser la question de savoir si les résultats auraient été différents si nous avions utilisé un appareil d'échographie polyvalent. Nous restons cependant convaincus du bien-fondé de ce type d'appareil totalement dédié à la pose des cathéters veineux centraux.

- le rapport coût/bénéfice n'a pas été étudié dans ce travail. 


\section{Conclusion}

La pose d'un cathéter veineux central en position jugulaire interne est un acte très fréquent dans nos services de réanimation. Les complications qui sont liées à la pose de ces cathéters sont fréquentes et coûteuses d'un point de vue médico-économique. Diminuer ces complications représente donc un défi de taille.

Dans cette lutte, l'échoguidage est un outil qui a fait ses preuves, mais dont l'utilisation tarde à s'imposer comme étant celle de référence. Cibler les populations à risque de complications, comme les patients obèses, pouvant bénéficier davantage de cette technique, pourrait être une approche pour ensuite généraliser son usage à l'ensemble de la population.

Dans ce sens, notre travail montre un bénéfice incontesté de l'échoguidage par rapport au simple repérage anatomique, dans la population de patients obèses en réanimation, sur le taux de complications liées à la pose d'un cathéter veineux central en position jugulaire interne, tout en facilitant la pose. 


\section{BIBLIOGRAPHIE}

1. Raad I. Intravascular-catheter-related infections. Lancet. 1998 Mar 21;351(9106):893898.

2. Vincent JL, Bihari DJ, Suter PM, Bruining HA, White J, Nicolas-Chanoin MH, et al. The prevalence of nosocomial infection in intensive care units in Europe. Results of the European Prevalence of Infection in Intensive Care (EPIC) Study. EPIC International Advisory Committee. JAMA. 1995 Aug 23-30;274(8):639-644.

3. Saarela E, Kari A, Nikki P, Rauhala V, Iisalo E, Kaukinen L. Current practice regarding invasive monitoring in intensive care units in Finland. A nationwide study of the uses of arterial, pulmonary artery and central venous catheters and their effect on outcome. The Finnish Intensive Care Study Group. Intensive Care Med. 1991;17(5):264-271.

4. Gordon AC, Saliken JC, Johns D, Owen R, Gray RR. US-guided puncture of the internal jugular vein: complications and anatomic considerations. J Vasc Interv Radiol. 1998 Mar-Apr;9(2):333-338.

5. Denys BG, Uretsky BF. Anatomical variations of internal jugular vein location: impact on central venous access. Crit Care Med. 1991 Dec;19(12):1516-1519.

6. Lichtenstein D, Saifi R, Augarde R, Prin S, Schmitt JM, Page B, et al. The Internal jugular veins are asymmetric. Usefulness of ultrasound before catheterization. Intensive Care Med. 2001 Jan;27(1):301-305.

7. Sulek CA, Blas ML, Lobato EB. A randomized study of left versus right internal jugular vein cannulation in adults. J Clin Anesth. 2000 Mar;12(2):142-145.

8. Maizel J, Slama M. Mise en place échoguidée des cathéters veineux centraux. Réanimation. 2013;22:196-203.

9. Lengelle F, Airapetian N, Maizel J, Samy Modeliar S, De Cagny B, Dupont H, et al. Comparaison de trois techniques de pose de cathéters veineux centraux. Ann Fr Anesth Réanim. 2008;27S(S83).

10. Randolph AG, Cook DJ, Gonzales CA, Pribble CG. Ultrasound guidance for placement of central venous catheters: a meta-analysis of the literature. Crit Care Med. 1996 Dec;24(12):2053-2058.

11. Bold RJ, Winchester DJ, Madary AR, Gregurich MA, Mansfield PF. Prospective, randomized trial of Doppler-assisted subclavian vein catheterization. Arch Surg. 1998 Oct;133(10):1089-1093. 
12. Feller-Kopman D. Ultrasound-guided internal jugular access: a proposed standardized approach and implications for training and practice. Chest. 2007 Jul;132(1):302-309.

13. Schummer W, Schummer C, Tuppatsch H, Fuchs J, Bloos F, Huttemann E. Ultrasound-guided central venous cannulation: is there a difference between Doppler and Bmode ultrasound? J Clin Anesth. 2006 May;18(3):167-172.

14. Karakitsos D, Labropoulos N, De Groot E, Patrianakos AP, Kouraklis G, Poularas J, et al. Real-time ultrasound-guided catheterisation of the internal jugular vein: a prospective comparison with the landmark technique in critical care patients. Critical care. 2006;10(6):R162.

15. Slama M, Novara A, Safavian A, Ossart M, Safar M, Fagon JY. Improvement of internal jugular vein cannulation using an ultrasound-guided technique. Intensive Care Med. 1997 Aug;23(8):916-919.

16. Airapetian N, Maizel J, Langelle F, Modeliar SS, Karakitsos D, Dupont H, et al. Ultrasound-guided central venous cannulation is superior to quick-look ultrasound and landmark methods among inexperienced operators: a prospective randomized study. Intensive Care Med. 2013 Nov;39(11):1938-1944.

17. Miller AH, Roth BA, Mills TJ, Woody JR, Longmoor CE, Foster B. Ultrasound guidance versus the landmark technique for the placement of central venous catheters in the emergency department. Academic emergency medicine : official journal of the Society for Academic Emergency Medicine. 2002 Aug;9(8):800-805.

18. Hilty WM, Hudson PA, Levitt MA, Hall JB. Real-time ultrasound-guided femoral vein catheterization during cardiopulmonary resuscitation. Ann Emerg Med. 1997 Mar;29(3):331-336; discussion 337.

19. Hrics P, Wilber S, Blanda MP, Gallo U. Ultrasound-assisted internal jugular vein catheterization in the ED. The American journal of emergency medicine. 1998 Jul;16(4):401403.

20. Hayashi H, Ootaki C, Tsuzuku M, Amano M. Respiratory jugular venodilation: its anatomic rationale as a landmark for right internal jugular vein puncture as determined by ultrasonography. Journal of cardiothoracic and vascular anesthesia. 2000 Aug;14(4):425-427.

21. Airapetian N, Samy Modeliar S, Lengelle F, Maizel J, De Cagny B, Dupont H, et al. Comparaison des trois téchniques de pose de cathéters veineux centraux. Réanimation. 2009;18S:S0044.

22. Nadig C, Leidig M, Schmiedeke T, Hoffken B. The use of ultrasound for the placement of dialysis catheters. Nephrol Dial Transplant. 1998 Apr;13(4):978-981. 
23. Milling TJ, Jr., Rose J, Briggs WM, Birkhahn R, Gaeta TJ, Bove JJ, et al. Randomized, controlled clinical trial of point-of-care limited ultrasonography assistance of central venous cannulation: the Third Sonography Outcomes Assessment Program (SOAP-3) Trial. Crit Care Med. 2005 Aug;33(8):1764-1769.

24. Lamperti M, Bodenham AR, Pittiruti M, Blaivas M, Augoustides JG, Elbarbary M, et al. International evidence-based recommendations on ultrasound-guided vascular access. Intensive Care Med. 2012 Jul;38(7):1105-1117.

25. Troianos CA, Hartman GS, Glas KE, Skubas NJ, Eberhardt RT, Walker JD, et al. Guidelines for performing ultrasound guided vascular cannulation: recommendations of the American Society of Echocardiography and the Society of Cardiovascular Anesthesiologists. J Am Soc Echocardiogr. 2011 Dec;24(12):1291-1318.

26. American Society of Anesthesiologists Task Force on Central Venous A, Rupp SM, Apfelbaum JL, Blitt C, Caplan RA, Connis RT, et al. Practice guidelines for central venous access: a report by the American Society of Anesthesiologists Task Force on Central Venous Access. Anesthesiology. 2012 Mar;116(3):539-573.

27. National Institute for Clinical Excellence. NICE technology appraisal guidance No.49: Guidance on the use of ultrasound locating devices for placing central venous catheters London: NICE; 2002. Available from: http://www.nice.org.uk/guidance/ta49.

28. McGrattan T, Duffty J, Green JS, O'Donnell N. A survey of the use of ultrasound guidance in internal jugular venous cannulation. Anaesthesia. 2008 Nov;63(11):1222-1225.

29. Bailey PL, Glance LG, Eaton MP, Parshall B, McIntosh S. A survey of the use of ultrasound during central venous catheterization. Anesth Analg. 2007 Mar;104(3):491-497.

30. Balls A, LoVecchio F, Kroeger A, Stapczynski JS, Mulrow M, Drachman D, et al. Ultrasound guidance for central venous catheter placement: results from the Central Line Emergency Access Registry Database. The American journal of emergency medicine. 2010 Jun;28(5):561-567.

31. Tovey G, Stokes M. A survey of the use of 2D ultrasound guidance for insertion of central venous catheters by UK consultant paediatric anaesthetists. Eur J Anaesthesiol. 2007 Jan;24(1):71-75.

32. Nguyen BV, Prat G, Vincent JL, Nowak E, Bizien N, Tonnelier JM, et al. Determination of the learning curve for ultrasound-guided jugular central venous catheter placement. Intensive Care Med. 2014 Jan;40(1):66-73. 
33. Bouaziz H, Zetlaoui PJ, Pierre S, Desruennes E, Fritsh N, Lapostolle F, et al. Guidelines on the use of ultrasound guidance for vascular access. Anaesth Crit Care Pain Med. 2015.

34. McGee DC, Gould MK. Preventing complications of central venous catheterization. N Engl J Med. 2003 Mar 20;348(12):1123-1133.

35. Wu SY, Ling Q, Cao LH, Wang J, Xu MX, Zeng WA. Real-time two-dimensional ultrasound guidance for central venous cannulation: a meta-analysis. Anesthesiology. 2013 Feb;118(2):361-375.

36. Schummer W, Schummer C, Rose N, Niesen WD, Sakka SG. Mechanical complications and malpositions of central venous cannulations by experienced operators. A prospective study of 1794 catheterizations in critically ill patients. Intensive Care Med. 2007 Jun;33(6):1055-1059.

37. Mermel LA. Prevention of intravascular catheter-related infections. Ann Intern Med. 2000 Mar 7;132(5):391-402.

38. Pronovost P, Needham D, Berenholtz S, Sinopoli D, Chu H, Cosgrove S, et al. An intervention to decrease catheter-related bloodstream infections in the ICU. N Engl J Med. 2006 Dec 28;355(26):2725-2732.

39. Merrer J, De Jonghe B, Golliot F, Lefrant JY, Raffy B, Barre E, et al. Complications of femoral and subclavian venous catheterization in critically ill patients: a randomized controlled trial. JAMA. 2001 Aug 8;286(6):700-707.

40. Deshpande KS, Hatem C, Ulrich HL, Currie BP, Aldrich TK, Bryan-Brown CW, et al. The incidence of infectious complications of central venous catheters at the subclavian, internal jugular, and femoral sites in an intensive care unit population. Crit Care Med. 2005 Jan;33(1):13-20; discussion 234-235.

41. Marik PE, Flemmer M, Harrison W. The risk of catheter-related bloodstream infection with femoral venous catheters as compared to subclavian and internal jugular venous catheters: a systematic review of the literature and meta-analysis. Crit Care Med. 2012 Aug;40(8):2479-2485.

42. Parienti JJ, Thirion M, Megarbane B, Souweine B, Ouchikhe A, Polito A, et al. Femoral vs jugular venous catheterization and risk of nosocomial events in adults requiring acute renal replacement therapy: a randomized controlled trial. JAMA. 2008 May 28;299(20):2413-2422.

43. Hirsch DR, Ingenito EP, Goldhaber SZ. Prevalence of deep venous thrombosis among patients in medical intensive care. JAMA. 1995 Jul 26;274(4):335-337. 
44. Cade JF. High risk of the critically ill for venous thromboembolism. Crit Care Med. $1982 \mathrm{Jul} ; 10(7): 448-450$.

45. Marik PE, Andrews L, Maini B. The incidence of deep venous thrombosis in ICU patients. Chest. 1997 Mar;111(3):661-664.

46. Shorr AF, Trotta RF, Alkins SA, Hanzel GS, Diehl LF. D-dimer assay predicts mortality in critically ill patients without disseminated intravascular coagulation or venous thromboembolic disease. Intensive Care Med. 1999 Feb;25(2):207-210.

47. Kollef MH, Zahid M, Eisenberg PR. Predictive value of a rapid semiquantitative Ddimer assay in critically ill patients with suspected venous thromboembolic disease. Crit Care Med. 2000 Feb;28(2):414-420.

48. Fraisse F, Holzapfel L, Couland JM, Simonneau G, Bedock B, Feissel M, et al. Nadroparin in the prevention of deep vein thrombosis in acute decompensated COPD. The Association of Non-University Affiliated Intensive Care Specialist Physicians of France. Am J Respir Crit Care Med. 2000 Apr;161(4 Pt 1):1109-1114.

49. Fabiani A, Pittiruti M, Russo S, Sanson G. Early-onset thrombosis of internal jugular vein associated with introducer catheter for heart catheterization in cardiac surgery. $\mathrm{J}$ Vasc Access. 2015 Feb 16;16(1):57-63.

50. Obésité. Available from: http://www.inserm.fr/thematiques/circulation-metabolismenutrition/dossiers-d-information/obesite.

51. Clinical Guidelines on the Identification, Evaluation, and Treatment of Overweight and Obesity in Adults--The Evidence Report. National Institutes of Health. Obes Res. 1998 Sep;6 Suppl 2:51S-209S.

52. Ray DE, Matchett SC, Baker K, Wasser T, Young MJ. The effect of body mass index on patient outcomes in a medical ICU. Chest. 2005 Jun;127(6):2125-2131.

53. Sakr Y, Madl C, Filipescu D, Moreno R, Groeneveld J, Artigas A, et al. Obesity is associated with increased morbidity but not mortality in critically ill patients. Intensive Care Med. 2008 Nov;34(11):1999-2009.

54. Goulenok C, Cariou A. Obesité en réaniamtion, définition, épidémiologie, pronostic. Réanimation. 2006;15:421-426.

55. Varon J, Marik P. Management of the obese critically ill patient. Crit Care Clin. 2001 Jan;17(1):187-200.

56. Marik P, Varon J. The obese patient in the ICU. Chest. 1998 Feb;113(2):492-498.

57. Seldinger SI. Catheter replacement of the needle in percutaneous arteriography; a new technique. Acta radiologica. 1953;39:368-376. 
58. Brun Buisson C, Rauss A, Legrand P. Semiquantitative culture of catheter tips. J Clin Microbiol. 1987;24:315-322.

59. Calle EE, Thun MJ, Petrelli JM, Rodriguez C, Heath CW, Jr. Body-mass index and mortality in a prospective cohort of U.S. adults. N Engl J Med. 1999 Oct 7;341(15):10971105.

60. Timsit JF. [Updating of the 12th consensus conference of the Societe de Reanimation de langue francaise (SRLF): catheter related infections in the intensive care unit]. Ann Fr Anesth Reanim. 2005 Mar;24(3):315-322.

61. McCabe WR, Jackson GR. Arch of Int Med. 1962;110:847-891.

62. Lan KKG, Demets DL. Discrete sequential boudaries for clinical trials. Biometrika. 1983 May;70(3):659-663.

63. O'Brien PC, Fleming TR. A multiple testing procedure for clinical trials. Biometrics. 1979 Sep;35(3):549-556.

64. Mallory DL, McGee WT, Shawker TH, Brenner M, Bailey KR, Evans RG, et al. Ultrasound guidance improves the success rate of internal jugular vein cannulation. A prospective, randomized trial. Chest. $1990 \mathrm{Jul}$;98(1):157-160.

65. Caridi JG, Hawkins IF, Jr., Wiechmann BN, Pevarski DJ, Tonkin JC. Sonographic guidance when using the right internal jugular vein for central vein access. AJR Am J Roentgenol. 1998 Nov;171(5):1259-1263.

66. Kwon TH, Kim YL, Cho DK. Ultrasound-guided cannulation of the femoral vein for acute haemodialysis access. Nephrol Dial Transplant. 1997 May;12(5):1009-1012.

67. Hind D, Calvert N, McWilliams R, Davidson A, Paisley S, Beverley C, et al. Ultrasonic locating devices for central venous cannulation: meta-analysis. BMJ. 2003 Aug $16 ; 327(7411): 361$.

68. Troianos CA, Jobes DR, Ellison N. Ultrasound-guided cannulation of the internal jugular vein. A prospective, randomized study. Anesth Analg. 1991 Jun;72(6):823-826.

69. Denys BG, Uretsky BF, Reddy PS. Ultrasound-assisted cannulation of the internal jugular vein. A prospective comparison to the external landmark-guided technique. Circulation. 1993 May;87(5):1557-1562.

70. Moon CH, Blehar D, Shear MA, Uyehara P, Gaspari RJ, Arnold J, et al. Incidence of posterior vessel wall puncture during ultrasound-guided vessel cannulation in a simulated model. Academic emergency medicine : official journal of the Society for Academic Emergency Medicine. 2010 Oct;17(10):1138-1141. 
71. Stone MB, Moon C, Sutijono D, Blaivas M. Needle tip visualization during ultrasound-guided vascular access: short-axis vs long-axis approach. The American journal of emergency medicine. 2010 Mar;28(3):343-347.

72. Chittoodan S, Breen D, O'Donnell BD, Iohom G. Long versus short axis ultrasound guided approach for internal jugular vein cannulation: a prospective randomised controlled trial. Medical ultrasonography. 2011 Mar;13(1):21-25.

73. Lorente L, Henry C, Martin MM, Jimenez A, Mora ML. Central venous catheterrelated infection in a prospective and observational study of 2,595 catheters. Critical care. 2005;9(6):R631-635.

74. Maecken T, Grau T. Ultrasound imaging in vascular access. Crit Care Med. 2007 May;35(5 Suppl):S178-185.

75. Mansfield PF, Hohn DC, Fornage BD, Gregurich MA, Ota DM. Complications and failures of subclavian-vein catheterization. N Engl J Med. 1994 Dec 29;331(26):1735-1738.

76. Eisen LA, Narasimhan M, Berger JS, Mayo PH, Rosen MJ, Schneider RF. Mechanical complications of central venous catheters. Journal of intensive care medicine. 2006 JanFeb;21(1):40-46.

77. Réseau RéaRaisin 2012. Available from: http://www.cclinparisnord.org.

78. Richards MJ, Edwards JR, Culver DH, Gaynes RP. Nosocomial infections in medical intensive care units in the United States. National Nosocomial Infections Surveillance System. Crit Care Med. 1999 May;27(5):887-892.

79. Flegal KM, Carroll MD, Ogden CL, Johnson CL. Prevalence and trends in obesity among US adults, 1999-2000. JAMA. 2002 Oct 9;288(14):1723-1727.

80. Bjorntorp P. Obesity. Lancet. 1997 Aug 9;350(9075):423-426.

81. El-Solh A, Sikka P, Bozkanat E, Jaafar W, Davies J. Morbid obesity in the medical ICU. Chest. 2001 Dec;120(6):1989-1997.

82. Goulenok C, Monchi M, Chiche JD, Mira JP, Dhainaut JF, Cariou A. Influence of overweight on ICU mortality: a prospective study. Chest. 2004 Apr;125(4):1441-1445.

83. Augoustides JG, Diaz D, Weiner J, Clarke C, Jobes DR. Current practice of internal jugular venous cannulation in a university anesthesia department: influence of operator experience on success of cannulation and arterial injury. Journal of cardiothoracic and vascular anesthesia. 2002 Oct;16(5):567-571.

84. Gualtieri E, Deppe SA, Sipperly ME, Thompson DR. Subclavian venous catheterization: greater success rate for less experienced operators using ultrasound guidance. Crit Care Med. 1995 Apr;23(4):692-697. 
85. Sekiguchi H, Tokita JE, Minami T, Eisen LA, Mayo PH, Narasimhan M. A prerotational, simulation-based workshop improves the safety of central venous catheter insertion: results of a successful internal medicine house staff training program. Chest. 2011 Sep;140(3):652-658.

86. Making Health Care Safer: A Critical Analysis of patient Safety Practices. Evidence Report/ Technology Assessment. 2001;43.

87. Matava C, Hayes J. A survey of ultrasound use by academic and community anesthesiologists in Ontario. Canadian journal of anaesthesia $=$ Journal canadien d'anesthesie. 2011 Oct;58(10):929-935.

88. Calvert N, Hind D, McWilliams R, Davidson A, Berverley CA, Thomas SM. Ultrasound for central venous cannulation: economic evaluation of cost-effectiveness. Anaesthesia. 2004;59:1116-1120. 


\section{ANNEXES}

Annexe 1. Echographe SiteRite V, Bard ${ }^{\circledR}$
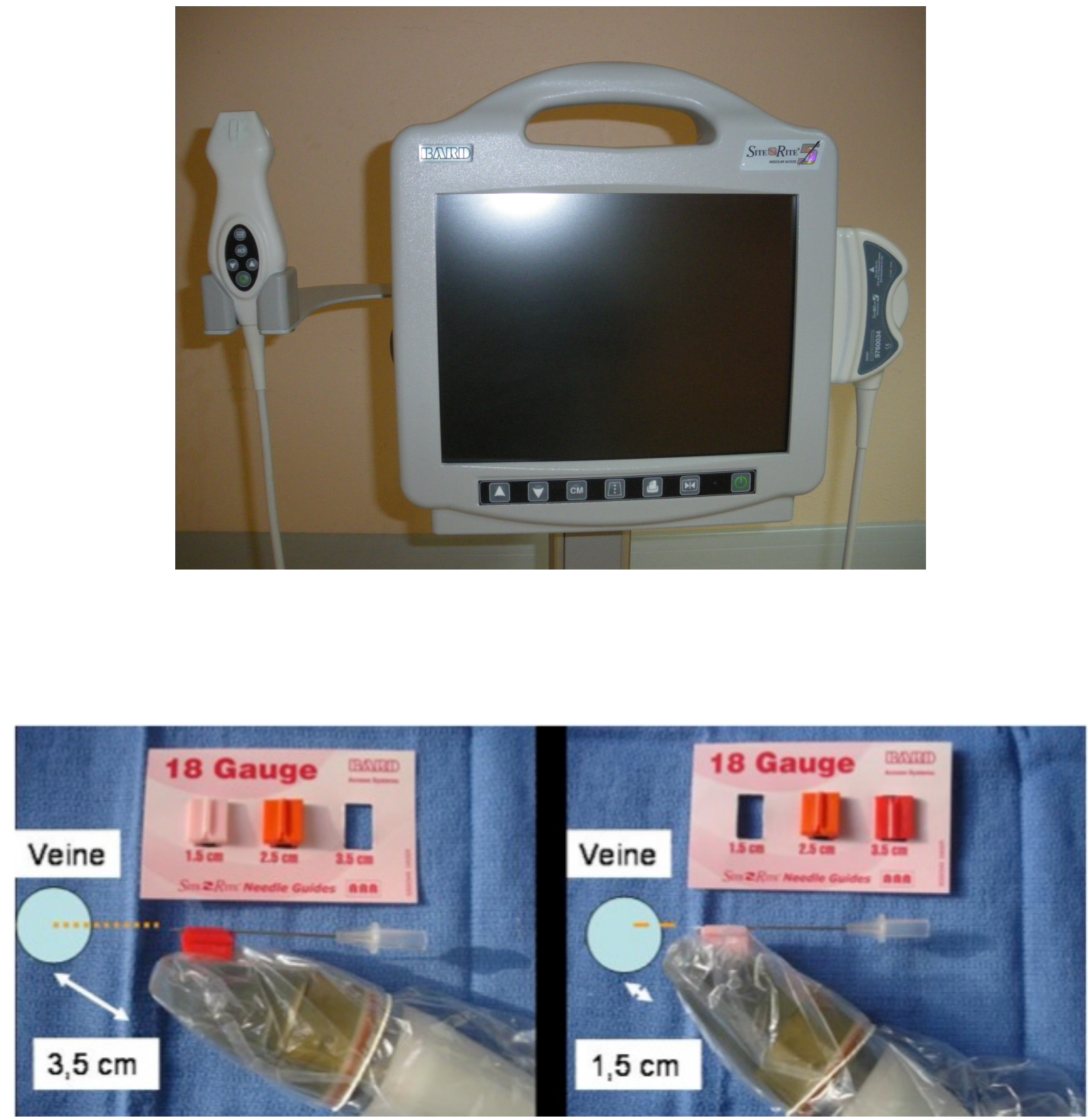
Annexe 2. Repérage et mesure de la profondeur de la veine (capture d'écran SiteRite V)

Exemple de visualisation de la veine jugulaire (compressible) et son rapport anatomique avec l'artère carotide interne. On note la graduation disponible permettant d'évaluer précisément la profondeur de la veine.

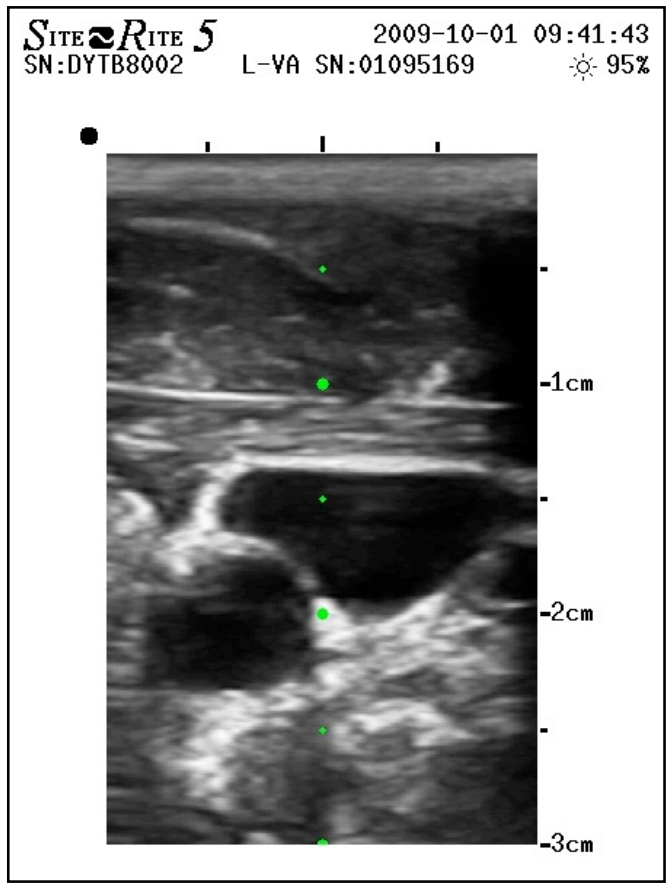




\section{Annexe 3. Définition de l'IGS II}

L'indice de gravité simplifié (IGS) II est un système simplifié d'évaluation de la gravité globale des patients hospitalisés en unité de soins intensifs (USI) ou en réanimation. Il est fondé sur la valeur des constantes physiologiques, l'âge, les antécédents et le type de pathologie. On utilise aujourd'hui la version II à 15 paramètres, qui permet de donner un score de gravité et de calculer un risque de décès hospitalier pour une équation de régression logistique. Cette version a été établie à partir d'une base de données nord-américaine et européenne de 13152 patients de 137 services de réanimation. Le score varie entre 0 et 194.

\begin{tabular}{|c|c|c|c|c|c|c|c|c|c|c|c|c|c|c|c|c|c|c|c|c|c|c|c|c|c|}
\hline Variable & $26 \mid$ & $13 \mid 1$ & 12 & 11 & 9 & 7 & 6 & 5 & 4 & \begin{tabular}{|l|l|}
3 & 2 \\
\end{tabular} & $\mathbf{0}$ & 1 & 2 & $3 \mid$ & 4 & 6 & 7 & 8 & 9 & 10 & 12 & 15 & 16 & 17 & 18 \\
\hline Âge (an) & & & & & & & & & & & 40 & & & & & & $\begin{array}{l}40- \\
59\end{array}$ & & & & 6069 & 7074 & 7579 & & $\begin{array}{l}> \\
80\end{array}$ \\
\hline $\begin{array}{l}\mathrm{FC}(\mathrm{b} \cdot \\
\min -1)\end{array}$ & & & & 40 & & & & & & & & & & & \begin{tabular}{|l|}
$120-$ \\
159
\end{tabular} & & $\begin{array}{l}> \\
160\end{array}$ & & & & & & & & \\
\hline $\begin{array}{l}\text { PAS } \\
(\mathrm{mmHg})\end{array}$ & & \begin{tabular}{|l|}
$>$ \\
70 \\
\end{tabular} & & & & & & 7099 & & & 100120 & & \begin{tabular}{|l|}
$>$ \\
200 \\
\end{tabular} & & & & & & & & & & & & \\
\hline $\mathrm{T}\left({ }^{\circ} \mathrm{C}\right)$ & & & & & & & & & & & $<39$ & & & $\begin{array}{l}> \\
39\end{array}$ & & & & & & & & & & & \\
\hline $\begin{array}{l}\mathrm{PaO} 2 / \mathrm{FI} \\
\mathrm{O} 2 \\
\mathrm{Si} \\
\mathrm{VA} / \mathrm{CPA} \\
\mathrm{P}\end{array}$ & & & & \begin{tabular}{l|l}
$<$ \\
100
\end{tabular} & $\begin{array}{l}100- \\
99\end{array}$ & & $\begin{array}{l}> \\
200\end{array}$ & & & & & & & & & & & & & & & & & & \\
\hline $\begin{array}{l}\text { Diurèse } \\
\mathrm{L} \cdot \mathrm{j}-1\end{array}$ & & & \begin{tabular}{l|l|}
$<$ & \\
0,5 & \\
\end{tabular} & & & & & & $\begin{array}{l}0,5- \\
0,99 \\
\end{array}$ & & 1 & & & & & & & & & & & & & & \\
\hline $\begin{array}{l}\text { Urée mmol } \\
\cdot \mathrm{L}-1 \mathrm{~g} \cdot \mathrm{L}-1\end{array}$ & & & & & & & & & & & $<100,6$ & & & & & \begin{tabular}{|l|}
$10-$ \\
$29,90,6-$ \\
1,79
\end{tabular} & & & & $>30>1,8$ & & & & & \\
\hline $\begin{array}{l}\text { Globules } \\
\text { blancs /1 } \\
000\end{array}$ & & & $\begin{array}{l}< \\
1,0\end{array}$ & & & & & & & & $1,0-19,9$ & & & $|>|$ & & & & & & & & & & & \\
\hline $\begin{array}{l}\text { Kaliémie } \\
\mathrm{mmol} \cdot-1\end{array}$ & & & & & & & & & & $<3$ & $3,0-4,9$ & & & $\begin{array}{l}7 \\
5\end{array}$ & & & & & & & & & & & \\
\hline \begin{tabular}{l|} 
Natrémie \\
$\mathrm{mmol} \cdot \mathrm{L}-1$
\end{tabular} & & & & & & & & \begin{tabular}{|l|}
$<$ \\
125
\end{tabular} & & & $125-144$ & 145 & & & & & & & & & & & & & \\
\hline $\begin{array}{l}\mathrm{HCO} 3 \\
\mathrm{mmol} \cdot \mathrm{L}-1\end{array}$ & & & & & & & $\begin{array}{l}< \\
15 \\
\end{array}$ & & & \begin{tabular}{|l|}
$15-$ \\
19
\end{tabular} & 20 & & & & & & & & & & & & & & \\
\hline $\begin{array}{l}\text { Bilirubine } \\
\mathrm{mmol} \cdot \mathrm{L}- \\
1 \mathrm{mg} \cdot \mathrm{L}-1\end{array}$ & & & & & & & & & & & $<68,4<40$ & & & & $\begin{array}{l}68,4 \\
102\end{array}$ & & & & $>102>60$ & & & & & & \\
\hline $\begin{array}{l}\text { Glasgow } \\
\text { (points) }\end{array}$ & \begin{tabular}{|l|l|l|l|l}
$<$ & 6 \\
6 & $a ̀$ \\
& & \\
\end{tabular} & & & & & \begin{tabular}{|l|l|}
9 \\
à \\
10 \\
\end{tabular} & & & & & $14-15$ & & & & & & & & & & & & & & \\
\hline $\begin{array}{l}\text { Maladie } \\
\text { schroniques }\end{array}$ & & & & & & & & & & & & & & & & & & & Métastasés & Hématologie & & & & sida & \\
\hline $\begin{array}{l}\text { Type } \\
\text { d'admission }\end{array}$ & & & & & & & & & & & \begin{tabular}{|l|} 
Chirurgie \\
programmée
\end{tabular} & & & & & Médical & & $\begin{array}{l}\text { Chirurgie } \\
\text { urgente }\end{array}$ & & & & & & & \\
\hline Total & & & & & & & & & & & & & & & & & & & & & & & & & \\
\hline
\end{tabular}




\section{Annexe 4. Echelle de Mac Cabe}

Cette classification tient compte de l'existence d'une maladie chronique sous-jacente et de l'appréciation de son pronostic dans les 3 derniers mois qui précèdent l'hospitalisation en réanimation. Ce regroupement très simple permet de distinguer des groupes à pronostic très différent. Le pronostic doit avoir été défini dans les trois mois précédant l'entrée dans le service de réanimation.

- 1 = Absence de maladie sous-jacente ou maladie n'affectant pas le pronostic vital.

- 2 = Maladies sous-jacentes mettant en jeu le pronostic vital à 5 ans (insuffisant cardiaque stade III, insuffisant respiratoire sous $0_{2}$ à domicile, cancer non métastasé, hypertension portale).

- 3 = Maladies sous-jacentes estimées fatales à 1 an (insuffisant cardiaque stade IV NYHA, insuffisant respiratoire déjà ventilé, cancer métastasé, décompensation hémorragique de cirrhose). 